\title{
Adaptive Coordinated Cooperative Control of Multi-Mobile Manipulators
}

\author{
Víctor H. Andaluz ${ }^{1}$, Paulo Leica ${ }^{2}$, \\ Flavio Roberti ${ }^{2}$, Marcos Toibero ${ }^{2}$ and Ricardo Carelli ${ }^{2}$ \\ ${ }^{1}$ Universidad Técnica de Ambato, \\ Facultad de Ingeniería en Sistemas, Electrónica e Industrial \\ ${ }^{2}$ Universidad Nacional de San Juan, \\ Instituto de Automática, \\ ${ }^{1}$ Ecuador \\ ${ }^{2}$ Argentina
}

\section{Introduction}

A coordinated group of robots can execute certain tasks, e.g. surveillance of large areas (Hougen et al., 2000), search and rescue (Jennings et al., 1997), and large objectstransportation (Stouten and De Graaf, 2004), more efficiently than a single specialized robot (Cao et al., 1997). Other tasks are simply not accomplishable by a single mobile robot, demanding a group of coordinated robots to perform it, like the problem of sensors and actuators positioning (Bicchi et al., 2008), and the entrapment/escorting mission (Antonelli et al., 2008). In such context, the term formation control arises, which can be defined as the problem of controlling the relative postures of the robots of a platoon that moves as a single structure (Consolini et al., 2007).

Mobile manipulator is nowadays a widespread term that refers to robots built by a robotic arm mounted on a mobile platform. This kind of system, which is usually characterized by a high degree of redundancy, combines the manipulability of a fixed-base manipulator with the mobility of a wheeled platform. Such systems allow the most usual missions of robotic systems which requiere both locomotion and manipulation abilities. Coordinated control of multiple mobile manipulators have attracted the attention of many researchers (Khatib et al., 1996; Fujii et al., 2007; Tanner et al., 2003; Yasuhisa et al., 2003). The interest in such systems stems from the capability for carrying out complex and dexterous tasks which cannot be simply made using a single robot. Moreover, multiple small mobile manipulators are also more appropriate for realizing several tasks in the human environments than a large and heavy mobile manipulator from a safety point of view.

Main coordination schemes for multiple mobile manipulators that can be found in the literature are:

1. Leader-follower control for mobile manipulator, where one or a group of mobile manipulators plays the role of a leader, which track a preplanned trajectory, and the 
rest of the mobile manipulators form the follower group which moves in conjunction with the leader mobile manipulators (Fujii et al., 2007; Hirata et al., 2004; Thomas et al., 2002). In Xin and Yangmin, 2006, a leader-follower type formation control is designed for a group of mobile manipulators. To overcome parameter uncertainty in the model of the robot, a decentralized control law is applied to individual robots, in which an adaptive $\mathrm{NN}$ is used to model robot dynamics online.

2. Hybrid position-force control by decentralized/centralized scheme, where the position of the object is controlled in a certain direction of the workspace and the internal force of the object is controlled in a small range of the origin (Khatib et al., 1996; Tanner et al., 2003; Yamamoto et al., 2004). In Zhijun et al., 2008, robust adaptive controllers of multiple mobile manipulators carrying a common object in a cooperative manner have been investigated with unknown inertia parameters and disturbances. At first, a concise dynamics consisting of the dynamics of mobile manipulators and the geometrical constraints between the end-effectors and the object is developed for coordinated multiple mobile manipulators. In Zhijun et al., 2009 coupled dynamics are presented for two cooperating mobile manipulators manipulating an object with relative motion in the presence of uncertainties and external disturbances. Centralized robust adaptive controllers are introduced to guarantee the motion and force trajectories of the constrained object. A simulation study to the decentralized dynamic control for a robot collective consisting of nonholonomic wheeled mobile manipulators is performed in Hao and Venkat, 2008, by tracking the trajectories of the load, where two reference signals are used for each robot, one for the mobile platform and another for end-effector of the manipulating arm.

To reduce performance degradation, on-line parameter adaptation is relevant in applications where the mobile manipulator dynamic parameters may vary, such as load transportation. It is also useful when the knowledge of the dynamic parameters is limited. As an example, the trajectory tracking task can be severely affected by the change imposed to the robot dynamics when it is carrying an object, as shown in (Martins et al., 2008). Hence, some formation control architectures already proposed in the literature have considered the dynamics of the mobile robots (Zhijun et al., 2008; Zhijun et al., 2009).

In this Chapter, it is proposed a novel method for centralized-decentralized coordinated cooperative control of multiple wheeled mobile manipulators. Also, it is worth noting that, differently to the work in Hao and Venkat, 2008, we use a single reference for the endeffector of the robot mobile manipulator.

Although centralized control approaches present intrinsic problems, like the difficulty to sustain the communication between the robots and the limited scalability, they have technical advantages when applied to control a group of robots with defined geometric formations. Therefore, there still exists significant interest in their use. As an example, in Antonelli et al., 2008, a centralized multi-robot system is proposed for an entrapment/escorting mission, where the escorted agent is kept in the centroid of a polygon of $n$ sides, surrounded by $n$ robots positioned in the vertices of the polygon. Another task for which it is important to keep a formation during navigation is large-objects transportation, since the load has a fixed geometric form. Another recent work dealing with centralized formation control is Mas et al., 2008, where a control approach based on a virtual structure, called Cluster Space Control, is presented. There, the positioning control is carried out considering the centroid of a geometric structure corresponding to a three-robot formation. 
In this Chapter, the proposed strategy conceptualizes the mobile manipulators system (with $n \geq 3$ ) as a single group, and the desired motions are specified as a function of cluster attributes, such as position, orientation, and geometry. These attributes guide the selection of a set of independent system state variables suitable for specification, control, and monitoring. The control is based on a virtual 3-dimensional structure, where the position control (or tracking control) is carried out considering the centroid of the upper side of a geometric structure (shaped as a prism) corresponding to a three-mobile manipulators formation. It is worth noting that in control problem formulating first it is considered three mobile manipulators robots, and then is generalized to mobile manipulators robots.

The proposed multi-layer control scheme is mainly divided in five modules: 1) the upper module is responsible for planning the trajectory to be followed by the team of mobile manipulators; 2) the next module controls the formation, whose shape is determined by the distance and angle between the end-effector of a mobile manipulator and the two other ones; 3 ) another module is responsible to generate the control signals to the end-effectors of the mobile manipulators, through the inverse kinematics of each robot. As a mobile manipulator is usually a redundant system, this redundancy can be used for the achievement of additional performances. In this layer two secondary objectives are considered: the avoidance of obstacles by the mobile platforms and the singular configuration prevention through the control of the system's manipulability; introduced by Yoshikawa (1985). 4) The adaptive dynamic compensation module compensates the dynamics of each mobile manipulator to reduce the velocity tracking error. It is worth noting that this controller has been designed based on a dynamic model having reference velocities as input signals. Also, it uses a robust updating law, which makes the dynamic compensation system robust to parameter variations and guarantees that no parameter drift will occur; 5) finally, the robots module represents the mobile manipulators.

It is worth noting that we propose a methodology to avoid obstacles in the trajectory of any mobile manipulator based on the concept of mechanical impedance of the interaction robots-environment, without deforming the virtual structure and maintaining its desired trajectory. It is considered that the obstacle is placed at a maximum height that it does not interfere with the workspace, so that the arm of the mobile manipulators can follow the desired trajectory even when the platform is avoiding the obstacle.

This Chapter is organized as follows. Section 2 shows the kinematic and dynamic models of the mobile manipulator. Section 3 presents the proposed multi-layer control scheme for the coordinated and cooperative control of mobile manipulators. While the forward and inverse kinematics transformations, necessary for the control scheme, are presented in Section 4. Section 5 describes the scalability for coordinated cooperative control of mobile manipulators. By its turn, Section 6 presents the design of the controller, and the analysis of the system's stability is developed. Next, simulation experiments results are presented and discussed in Section 7, and finally the Chapter conclusions are given in Section 8.

\section{Mobile manipulator models}

The mobile manipulator configuration is defined by a vector $\mathbf{q}$ of $n$ independent coordinates, called generalized coordinates of the mobile manipulator, where $\mathbf{q}=\left[\begin{array}{llll}q_{1} & q_{2} & \cdots & q_{n}\end{array}\right]^{T}=\left[\begin{array}{ll}\mathbf{q}_{p}^{T} & \mathbf{q}_{a}^{T}\end{array}\right]^{T}$ where $\mathbf{q}_{a}$ represents the generalized coordinates of the 
arm, and $\mathbf{q}_{p}$ the generalized coordinates of the mobile platform. We notice that $n=n_{a}+n_{p}$, where $n_{a}$ and $n_{p}$ are respectively the dimensions of the generalized spaces associated to the robotic arm and to the mobile platform. The configuration $\mathbf{q}$ is an element of the mobile manipulator configuration space; denoted by $\mathcal{N}$. The location of the end-effector of the mobile manipulator is given by the $m$-dimensional vector $\mathbf{h}=\left[\begin{array}{llll}h_{1} & h_{2} & \ldots & h_{m}\end{array}\right]^{T}$ which define the position and the orientation of the end-effector of the mobile manipulator in $\mathcal{R}$. Its $m$ coordinates are the operational coordinates of the mobile manipulator. The set of all locations constitutes the mobile manipulator operational space, denoted by $\mathcal{M}$.

The location of the mobile manipulator end-effector can be defined in different ways according to the task, i.e., it can be considered only the position of the end-effector or both its position and its orientation.

\subsection{Mobile manipulator kinematic model}

The kinematic model of a mobile manipulator gives the location of the end-effector $\mathbf{h}$ as a function of the robotic arm configuration and the platform location (or its operational coordinates as functions of the robotic arm generalized coordinates and the mobile platform operational coordinates).

$$
\begin{gathered}
f: \mathcal{N}_{a} \times \mathcal{M}_{p} \rightarrow \mathcal{M} \\
\left(\mathbf{q}_{\mathrm{a}}, \mathbf{q}_{\mathrm{p}}\right) \mapsto \mathbf{h}=f\left(\mathbf{q}_{\mathrm{a}}, \mathbf{q}_{\mathrm{p}}\right)
\end{gathered}
$$

where, $\mathcal{N}_{a}$ is the configuration space of the robotic arm, $\mathcal{M}_{p}$ is the operational space of the platform.

The instantaneous kinematic model of a mobile manipulator gives the derivative of its endeffector location as a function of the derivatives of both the robotic arm configuration and the location of the mobile platform,

$$
\dot{\mathbf{h}}=\frac{\partial f}{\partial \mathbf{q}}\left(\mathbf{q}_{a}, \mathbf{q}_{p}\right) \mathbf{v}
$$

where, $\dot{\mathbf{h}}=\left[\begin{array}{llll}\dot{h}_{1} & \dot{h}_{2} & \ldots & \dot{h}_{m}\end{array}\right]^{T}$ is the vector of the end-effector velocity, $\mathbf{v}=\left[\begin{array}{llll}v_{1} & v_{2} & \ldots & v_{\delta_{n}}\end{array}\right]^{T}$ $=\left[\begin{array}{ll}\mathbf{v}_{\mathrm{p}}^{\mathrm{T}} & \mathbf{v}_{\mathrm{a}}^{\mathrm{T}}\end{array}\right]^{T}$ is the control vector of mobility of the mobile manipulator. Its dimension is $\delta_{n}=\delta_{n p}+\delta_{n a}$, where $\delta_{n p}$ and $\delta_{n a}$ are respectively the dimensions of the control vector of mobility associated to the mobile platform and to the robotic arm. Now, after replacing $\mathbf{J}(\mathbf{q})=\frac{\partial f}{\partial \mathbf{q}}\left(\mathbf{q}_{\mathrm{a}}, \mathbf{q}_{\mathrm{p}}\right) \mathbf{T}(\mathbf{q})$ in the above equation, we obtain

$$
\dot{\mathbf{h}}(t)=\mathbf{J}(\mathbf{q}) \mathbf{v}(t)
$$


where, $\mathbf{J}(\mathbf{q})$ is the Jacobian matrix that defines a linear mapping between the vector of the mobile manipulator velocities $\mathbf{v}(t)$ and the vector of the end-effector velocity $\dot{\mathbf{h}}(t)$, and $\mathbf{T}(\mathbf{q})$ is the transformation matrix that relates joints velocities $\dot{\mathbf{q}}(t)$ and mobile manipulator velocities $\mathbf{v}(t)$ such that $\dot{\mathbf{q}}(t)=\mathbf{T}(\mathbf{q}) \mathbf{v}(t)$.

Remark 1: The transformation matrix $\mathbf{T}(\mathbf{q})$ includes the non-holonomic constraints of the mobile platform.

The Jacobian matrix is, in general, a function of the configuration $\mathbf{q}$; those configurations at which $\mathbf{J}(\mathbf{q})$ is rank-deficient are termed singular kinematic configurations. It is fundamental to notice that, in general, the dimension of the operational space $m$ is less than the degree of mobility of the mobile manipulator, therefore the system is redundant.

\subsection{Mobile manipulator dynamic model}

The mathematic model that represents the dynamics of a mobile manipulator can be obtained from Lagrange's dynamic equations, which are based on the difference between the kinetic and the potential energy of each of the joints of the robot (energy balance) (Spong and Vidyasagar, 1989; Yoshikawa, 1990; Sciavicco and Siciliano, 2000). The dynamic equation of the mobile manipulator can be represented as follows,

$$
\overline{\mathbf{M}}(\mathbf{q}) \dot{\mathbf{v}}+\overline{\mathbf{C}}(\mathbf{q}, \mathbf{v}) \mathbf{v}+\overline{\mathrm{G}}(\mathbf{q})+\overline{\mathbf{d}}=\overline{\mathbf{B}}(\mathbf{q}) \mathbf{\tau}
$$

where, $\mathbf{q}=\left[q_{1}, \ldots, q_{n}\right]^{T} \in \mathfrak{R}^{n}$ is the general coordinate system vector of the mobile manipulator, $\mathbf{v}=\left[v_{1}, \ldots, v_{\delta_{n}}\right]^{T} \in \mathfrak{R}^{\delta_{n}}$ is the velocity vector of the mobile manipulator, $\overline{\mathbf{M}}(\mathbf{q}) \in \mathfrak{R}^{\delta_{n} \times \delta_{n}}$ is a symmetrical positive definite matrix that represents the system's inertia, $\overline{\mathbf{C}}(\mathbf{q}, \mathbf{v}) \mathbf{v} \in \mathfrak{R}^{\delta_{n}}$ represents the components of the centripetal and Coriolis forces, $\overline{\mathbf{G}}(\mathbf{q}) \in \mathfrak{R}^{\delta_{n}}$ represents the gravitational forces, $\overline{\mathbf{d}}$ denotes bounded unknown disturbances including the unmodeled dynamics, $\mathbf{\tau} \in \mathfrak{R}^{\delta_{n}}$ is the torque input vector, $\overline{\mathbf{B}}(\mathbf{q}) \in \mathfrak{R}^{\delta_{n} \times \delta_{n}}$ is the transformation matrix of the control actions.

Most of the commercially available robots have low level PID controllers in order to follow the reference velocity inputs, thus not allowing controlling the voltages of the motors directly. Therefore, it becomes useful to express the dynamic model of the mobile manipulator in a more appropriate way, taking the rotational and longitudinal reference velocities as the control signals. To do so, the velocity servo controller dynamics are included in the model. The dynamic model of the mobile manipulator, having as control signals the reference velocities of the system, can be represented as follows,

$$
\mathbf{M}(\mathbf{q}) \dot{\mathbf{v}}+\mathrm{C}(\mathbf{q}, \mathbf{v}) \mathbf{v}+\mathrm{G}(\mathbf{q})+\mathrm{d}=\mathbf{v}_{\text {ref }}
$$


where $\mathbf{M}(\mathbf{q})=\mathbf{H}^{-\mathbf{1}}(\overline{\mathbf{M}}+\mathbf{D}), \mathbf{C}(\mathbf{q}, \mathbf{v})=\mathbf{H}^{-\mathbf{1}}(\overline{\mathbf{C}}+\mathbf{P}), \mathbf{G}(\mathbf{q})=\mathbf{H}^{-\mathbf{1}} \overline{\mathbf{G}}(\mathbf{q})$ and $\mathbf{d}=\mathbf{H}^{-\mathbf{1}} \overline{\mathbf{d}}$. Thus, $\mathbf{M}(\mathbf{q}) \in \mathfrak{R}^{\delta_{n} \times \delta_{n}}$ is a positive definite matrix, $\mathbf{C}(\mathbf{q}, \mathbf{v}) \mathbf{v} \in \mathfrak{R}^{\delta_{n}}, \mathbf{G}(\mathbf{q}) \in \mathfrak{R}^{\delta_{n}}, \mathbf{d} \in \mathfrak{R}^{\delta_{n}}$ and $\mathbf{v}_{\text {ref }} \in \mathfrak{R}^{\delta_{n}}$ is the vector of velocity control signals, $\mathbf{H} \in \mathfrak{R}^{\delta_{n} \times \delta_{n}}, \mathbf{D} \in \mathfrak{R}^{\delta_{n} \times \delta_{n}}$ and $\mathbf{P} \in \mathfrak{R}^{\delta_{n} \times \delta_{n}}$ are positive definite constant diagonal matrices containing the physical parameters of the mobile manipulator, motors, and velocity controllers of both the mobile platform and the manipulator. It is important to remark that $\mathbf{H}, \mathbf{D}$ and $\mathbf{P}$ are positive definite constant diagonal matrices, hence the properties for the dynamic model with reference velocities as control signals (3) were obtained on based of the properties of the dynamic model (2):

Property 1. Matrix $\mathbf{M}(\mathbf{q})$ is positive definite, additionally it is known that

$$
\|\mathbf{M}(\mathbf{q})\|<k_{M}
$$

Property 2. Furthermore, the following inequalities are also satisfied

$$
\|\mathbf{C}(\mathbf{q}, \mathbf{v})\|<k_{c}\|\mathbf{v}\|
$$

Property 3. Vector $\mathbf{G}(\mathbf{q})$ and $\mathbf{d}$ are bounded

$$
\|\mathbf{G}(\mathbf{q})\|<k_{G} ; \quad\|\mathbf{d}\|<k_{d}
$$

where, $k_{c}, k_{M}, k_{G}$ and $k_{d}$ denote some positive constants.

Property 4. The dynamic model of the mobile manipulator can be represented by

$$
M(q) \dot{v}+C(q, v) v+G(q)+d=\Phi(q, v) X
$$

where, $\boldsymbol{\Phi}(\mathbf{q}, \mathbf{v}) \in \mathfrak{R}^{\delta_{n} \times l}$ and $\boldsymbol{X}=\left[\begin{array}{llll}\chi_{1} & \chi_{2} & \cdots & \chi_{l}\end{array}\right]^{T}$ is the vector of $l$ unknown parameters of the mobile manipulator, i.e., mass of the mobile robot, mass of the robotic arm, physical parameters of the mobile manipulator, motors, velocity, etc.

For the sake of simplicity, from now on it will be written $\mathbf{M}=\mathbf{M}(\mathbf{q}), \mathbf{C}=\mathbf{C}(\mathbf{q}, \mathbf{v})$ and $\mathbf{G}=\mathbf{G}(\mathbf{q})$.

Hence, the full mathematical model of the mobile manipulator robot is represented by (1), the instantaneous kinematic model and (3), the dynamic model, taking the reference velocities of the system as input signals.

\section{Multi-layers control scheme}

Figure 1, shows the Multi-layer control Scheme of the coordinated cooperative control of mobile manipulators which is taken into account in this Chapter.

Each layer works as an independent module, dealing with a specific part of the problem of coordinated cooperative control, and such control scheme includes a basic structure defined 
by the formation control layer, the kinematic control layer, the robots layer and the environment layer.

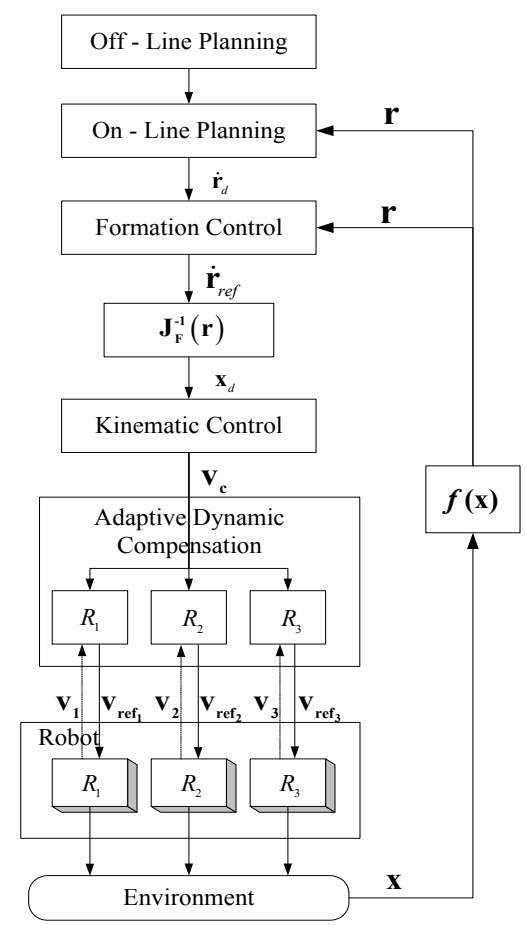

Fig. 1. Multi-layer control scheme

- The Off-line Planning layer is responsible for setting up the initial conditions, thus generating the trajectory of the object to be tracked, and for establishing the desired structure. The On-line Planning layer is capable of changing the references in order to make the formation to react to the environment, e.g., to modify the trajectory to avoid obstacles (it should be included only when a centralized obstacle avoidance strategy is considered; in this work it is considered the decentralized obstacle avoidance).

- $\quad$ The Formation Control layer is responsible for generating the control signals to be sent to the mobile manipulators, working as a team, in order to reach the desired values established by the planning layers.

- The Kinematic Control layer is responsible for generating the control signals to the endeffector of the mobile manipulators considering different control objectives.

- $\quad$ The Adaptive Dynamic Compensation layer compensates the dynamics of each robot to reduce the velocity tracking error.

- The Robot layer represents the mobile manipulators (mobile manipulators with unicyclelike mobile platforms, car-like and/or omnidirectional type), and finally;

- The Environment layer represents all objects surrounding the mobile manipulators, including the mobile manipulators themselves, with their external sensing systems, necessary for implementing obstacle avoidance. 
One of the main advantages of the proposed scheme is the independence of each layer, i.e., changes within a layer do not cause structural changes in the other layers. As an example, several kinematic controllers or dynamic compensation approaches can be tested using the same formation control strategy and vice-versa. It is worth mentioning that a simple structure can be obtained from the presented scheme, that is, some layers can be eliminated whenever the basic structure is maintained and the absence of the eliminated layers do not affect the remaining layers. For example, the On-line Planning layer could be discarded in the case of trajectory tracking or path following by a multirobot formation in a known environment free of obstacles, because the entire task accomplishment is controlled by the Formation Control layer. Also the Adaptive Dynamic Compensation layer can be suppressed, for applications demanding low velocities and light load transportation.

On the other hand, it is important to stress that some additional blocks are necessary to complete the multi-layer scheme, such as $\mathbf{J}_{\mathbf{F}}^{-1}(\mathbf{r})$ and $f(\mathbf{x})$, which represents the inverse formation Jacobian matrix, and the forward kinematic transformation function for the formation, respectively.

Remark 2: The mobile manipulators can be different, i.e., each mobile manipulator can be built by different types mobile platforms or/and different types robotic arms. Thus each mobile manipulator has its own configuration.

Remark 3: A mobile manipulator is defined as a redundant system because it has more degrees of freedom than required to achieve the desired end-effector motion. Hence, the redundancy of such systems can be effectively used for the achievement of additional performances.

\section{Kinematic transformation}

The proposed coordinated cooperative control method considers three or more mobile manipulators. In the first step, only three mobile manipulators are considered. In this case the control method is based on creating a regular or irregular prism defined by the position of the end-effector of each mobile manipulator. The location of the upper side of the prism in the plane $X-Y$ of the global framework is defined by $\mathbf{P}_{\mathbf{F}}=\left[\begin{array}{lll}x_{F} & y_{F} & \psi_{F}\end{array}\right]$, where $\left(x_{F}, y_{F}\right)$ represents the position of its centroid, and $\psi_{F}$ represents its orientation with respect to the global $Y$-axis. The structure shape of the prism (regular or irregular) is defined by $\mathbf{S}_{\mathbf{F}}=\left[\begin{array}{llllll}p_{F} & q_{F} & \beta_{F} & z_{1 F} & z_{2 F} & z_{3 F}\end{array}\right]$, where, $p_{F}$ represents the distance between $\mathbf{h}_{1}$ and $\mathbf{h}_{2}$, $q_{F}$ the distance between $\mathbf{h}_{1}$ and $\mathbf{h}_{3}, \beta_{F}$ the angle formed by $\mathbf{h}_{2} \hat{\mathbf{h}}_{1} \mathbf{h}_{3}$ and $\left(z_{1 F}, z_{2 F}, z_{3 F}\right)$ represents the height of the upper side of the prism. This situation is illustrated in Figure 2.

Remark 4: $\mathbf{h}_{i}$ represents the position the end-effector of the $i$-th mobile manipulator.

The relationship between the prism pose-orientation-shape and the end-effector positions of the mobile manipulators is given by the forward and inverse kinematics transformation, i.e., $\mathbf{r}=f(\mathbf{x})$ and $\mathbf{x}=f^{-1}(\mathbf{r})$, where $\mathbf{r}=\left[\begin{array}{ll}\mathbf{P}_{\mathbf{F}} & \mathbf{S}_{\mathbf{F}}\end{array}\right]^{T}$ and $\mathbf{x}=\left[\begin{array}{lll}\mathbf{h}_{1}^{T} & \mathbf{h}_{2}^{T} & \mathbf{h}_{3}^{T}\end{array}\right]^{T}$. 


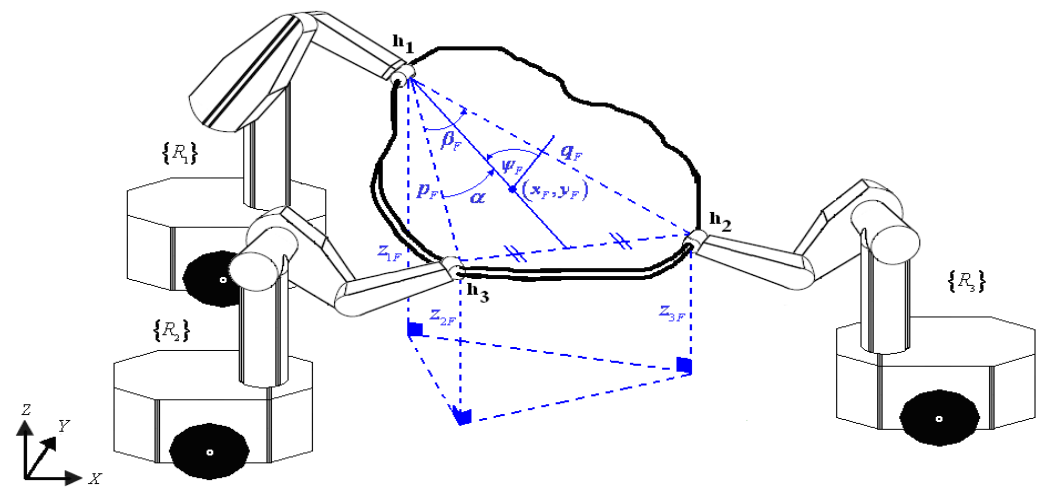

Fig. 2. Structure variables

The forward kinematic transformation $f($.$) , as shown in figure 2, is given by$

$$
\mathbf{P}_{\mathbf{F}}=\left[\begin{array}{c}
\frac{x_{1}+x_{2}+x_{3}}{3} \\
\frac{y_{1}+y_{2}+y_{3}}{3} \\
\arctan \frac{\frac{2}{3} x_{1}-\frac{1}{3}\left(x_{2}+x_{3}\right)}{\frac{2}{3} y_{1}-\frac{1}{3}\left(y_{2}+y_{3}\right)}
\end{array}\right]^{T} ; \quad \mathbf{S}_{\mathbf{F}}=\left[\begin{array}{c}
\sqrt{\left(x_{1}-x_{2}\right)^{2}+\left(y_{1}-y_{2}\right)^{2}} \\
\sqrt{\left(x_{1}-x_{3}\right)^{2}+\left(y_{1}-y_{3}\right)^{2}} \\
\arccos \frac{p_{F}^{2}+q_{F}^{2}-r_{F}^{2}}{2 p_{F} q_{F}} \\
z_{1 F} \\
z_{2 F} \\
z_{3 F}
\end{array}\right]^{T}
$$

where, $r_{F}=\sqrt{\left(x_{2}-x_{3}\right)^{2}+\left(y_{2}-y_{3}\right)^{2}}$. In turn, for the inverse kinematic transformation $f^{-1}($.$) , two representations are possible, depending on the disposition of the mobile$ manipulators in the prism shape (clockwise or counter-clockwise). Such disposition can be referred to as $R_{1} R_{2} R_{3}$ or $R_{1} R_{3} R_{2}$ sequence ( $R_{i}$ represents the $\mathrm{i}$-th mobile manipulator robot). Considering the first possibility, $\mathbf{x}=f_{R_{1} R_{2} R_{3}}^{-1}(\mathbf{r})$ is given by,

$$
\mathbf{x}=\left[\begin{array}{c}
\mathbf{h}_{1} \\
\mathbf{h}_{2} \\
\mathbf{h}_{3}
\end{array}\right]=\left[\begin{array}{c}
x_{F}+\frac{2}{3} h_{F} \sin \psi_{F} \\
y_{F}+\frac{2}{3} h_{F} \cos \psi_{F} \\
z_{1 F} \\
x_{F}+\frac{2}{3} h_{F} \sin \psi_{F}-p_{F} \sin \left(\alpha+\psi_{F}\right) \\
y_{F}+\frac{2}{3} h_{F} \cos \psi_{F}-p_{F} \cos \left(\alpha+\psi_{F}\right) \\
z_{2 F} \\
x_{F}+\frac{2}{3} h_{F} \sin \psi_{F}+q_{F} \sin \left(\beta_{F}-\alpha-\psi_{F}\right) \\
y_{F}+\frac{2}{3} h_{F} \cos \psi_{F}-q_{F} \cos \left(\beta_{F}-\alpha-\psi_{F}\right) \\
z_{3 F}
\end{array}\right]
$$


where, $h_{F}=\sqrt{\frac{1}{2}\left(p_{F}^{2}+q_{F}^{2}-\frac{1}{2} r_{F}^{2}\right)}$ represents the distance between the end-effector $\mathbf{h}_{1}$ and the point in the middle of the segment $\overline{\mathbf{h}_{2} \mathbf{h}_{3}}$, passing through $\left(x_{F}, y_{F}\right)$, and $\alpha=\arccos \frac{p_{F}^{2}+h_{F}^{2}-\frac{1}{4} r_{F}^{2}}{2 p_{F} h_{F}}$. On the other hand, $\mathbf{x}=f_{R_{1} R_{3} R_{2}}^{-1}(\mathbf{r})$ is given by

$$
\mathbf{x}=\left[\begin{array}{c}
\mathbf{h}_{1} \\
\mathbf{h}_{2} \\
\mathbf{h}_{3}
\end{array}\right]=\left[\begin{array}{c}
x_{F}+\frac{2}{3} h_{F} \sin \psi_{F} \\
y_{F}+\frac{2}{3} h_{F} \cos \psi_{F} \\
z_{1 F} \\
x_{F}+\frac{2}{3} h_{F} \sin \psi_{F}+p_{F} \sin \left(\alpha-\psi_{F}\right) \\
y_{F}+\frac{2}{3} h_{F} \cos \psi_{F}-p_{F} \cos \left(\alpha-\psi_{F}\right) \\
z_{2 F} \\
x_{F}+\frac{2}{3} h_{F} \sin \psi_{F}-q_{F} \sin \left(\beta_{F}-\alpha+\psi_{F}\right) \\
y_{F}+\frac{2}{3} h_{F} \cos \psi_{F}-q_{F} \cos \left(\beta_{F}-\alpha+\psi_{F}\right) \\
z_{3 F}
\end{array}\right]
$$

Figure 3 shows the control structure proposed in this Chapter for the coordinated cooperative control of mobile manipulators. Taking the time derivative of the forward and the inverse kinematics transformations we can obtain the relationship between the time variations of $\mathbf{x}(t)$ and $\mathbf{r}(t)$, represented by the Jacobian matrix $\mathbf{J}_{\mathbf{F}}$, which is given by

$$
\dot{\mathbf{r}}=\mathrm{J}_{\mathbf{F}}(\mathbf{x}) \dot{\mathbf{x}}
$$

and in the inverse way is given by

$$
\dot{\mathbf{x}}=\mathrm{J}_{\mathrm{F}}^{-1}(\mathbf{r}) \dot{\mathbf{r}}
$$

where,

$$
\mathbf{J}_{\mathbf{F}}(\mathbf{x})=\frac{\partial \mathbf{r}_{f \times 1}}{\partial \mathbf{x}_{e \times 1}} \quad \text { and } \quad \mathbf{J}_{\mathbf{F}}^{-1}(\mathbf{r})=\frac{\partial \mathbf{x}_{e \times 1}}{\partial \mathbf{r}_{f \times 1}} \quad \text { with } e, f=1,2 . ., 9
$$

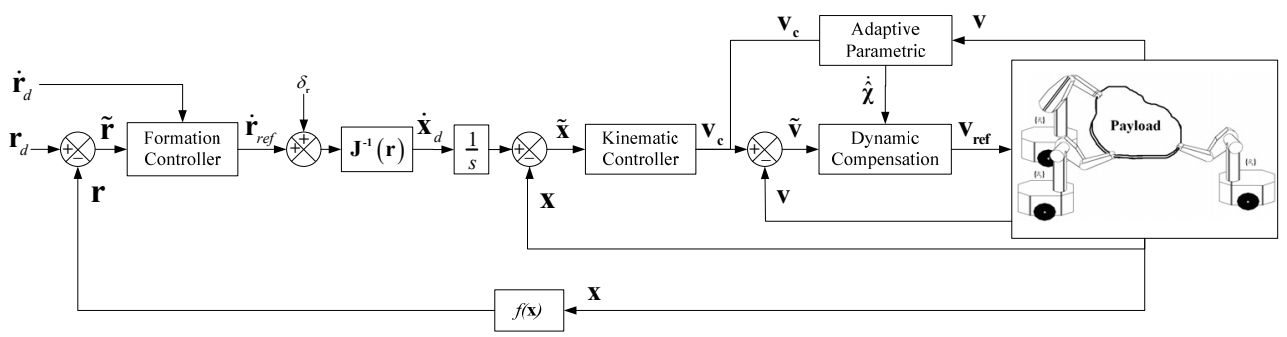

Fig. 3. Control system block diagram 


\section{Scalability for the cooperative control of multi-mobile manipulator}

This Subsection proposes a way to generalize the control system associated to the coordinated cooperation of three mobile manipulators (virtual structure prism) to a coordinated cooperation of $n>3$ mobile manipulators. Such proposition is based on the decomposition of a virtual 3-dimensional structure of $n$ vertices into simpler components, i.e., $n-2$ prisms. The idea is to take advantage of the control scheme proposed for a virtual prism to implement a coordinated cooperative control of $n>3$ mobile manipulators using the same kinematics transformations presented in previous Section 3, thus not demanding to change the Jacobian (Figure 4).

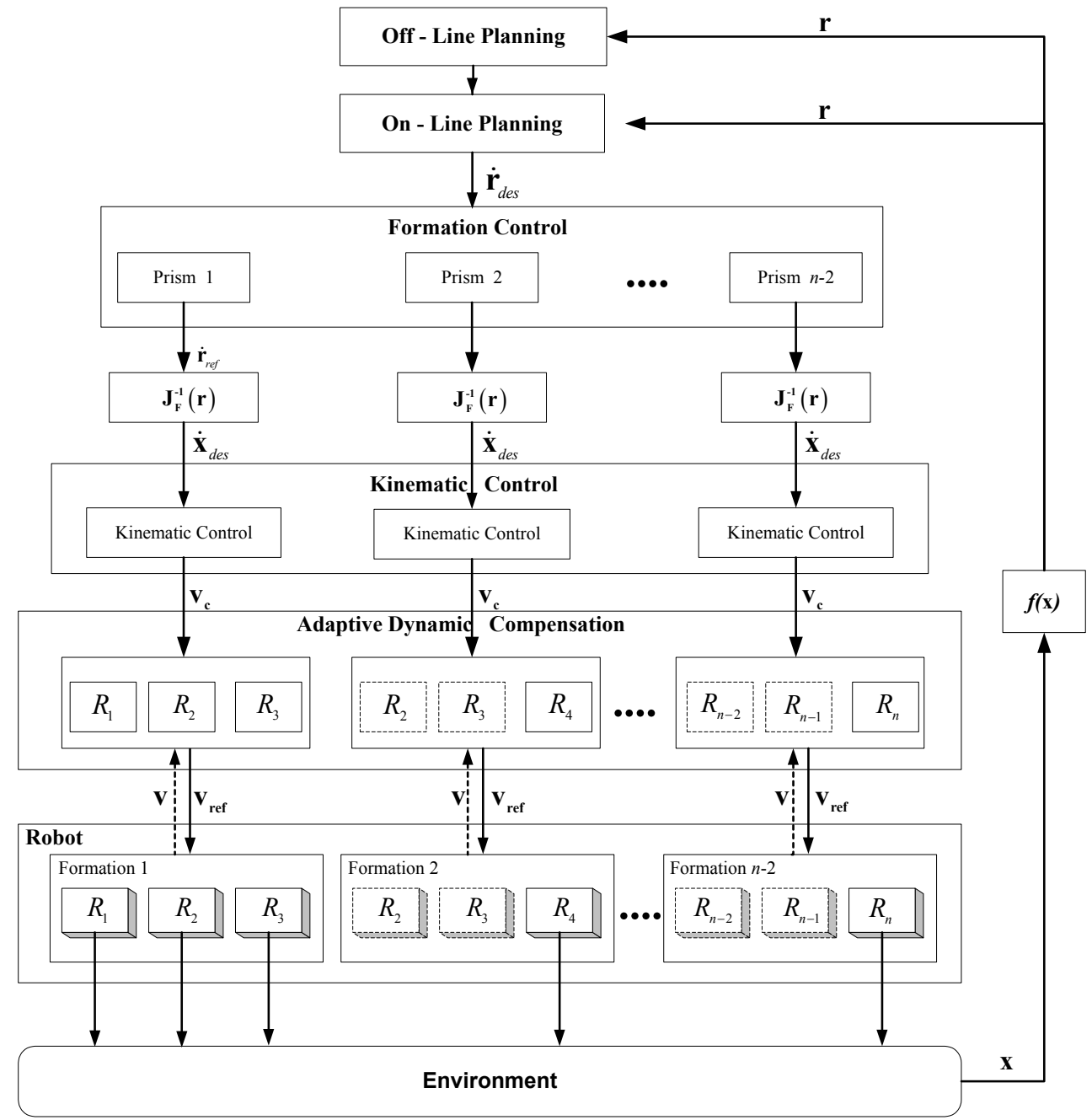

Fig. 4. Scalability in the multi-layer control 
To do that, one should first label the mobile manipulators $R_{i}, i=1,2,3 \ldots, n$ and determine the leader prism of the whole formation $\left(R_{2} R_{1} R_{3}\right.$ or $R_{3} R_{1} R_{2}$, paying attention to the sequence $\mathbf{A B C}$ or $\mathbf{A C B})$. After that, new prisms are formed with the remaining mobile manipulators, based on a simple algorithm: a new prism is formed with the last two mobile manipulators robots of the last prism already formed and the next mobile manipulator in the list of labelled mobile manipulators (in other words, $R_{j+1} R_{j} R_{j+2}$ or $R_{j+2} R_{j} R_{j+1}$ where $j=1,2, \ldots, n-2$ represents the current virtual structure prism). Additionally, from previous Section 6.3, a set of desired virtual structure variables $S_{F_{j}}=\left[\begin{array}{llllll}p_{F_{j}} & q_{F_{j}} & \beta_{F_{j}} & z_{1 F_{j}} & z_{2 F_{j}} & z_{3 F_{j}}\end{array}\right]$ is assigned to each virtual structure prism. Actually, the number of virtual structure variables is the same, but three of the variables has its value defined by the previous formation, i.e., $2(n-2)+1$, instead of $3(n-2)$, because it is assumed that $S_{F_{j}}=\left[\begin{array}{llllll}p_{F_{j-1}} & q_{F_{j}} & \beta_{F_{j}} & z_{1 F_{j-1}} & z_{2 F_{j-1}} & z_{3 F_{j}}\end{array}\right]$.

One point that deserves to be mentioned here is the control signals generated: there will always be a redundancy in the virtual structures with more than three mobile manipulators. For example, the mobile manipulators $R_{2}$ and $R_{3}$, in a virtual structures of four mobile manipulators, will receive control signals associated to the errors of the two virtual prisms ( $R_{2} R_{1} R_{3}$ and $R_{3} R_{2} R_{4}$, for example). In this work, however, the implementation chosen is one in which the mobile manipulators $R_{j+2}$ will receive control signals only from the controllers associated to the $j \geq 2$ virtual prisms, while the mobile manipulators $R_{1}, R_{2}$ and $R_{3}$ will receive the signals generated by the controller associated to the leader prism $(j=1)$.

Remark 5: the proposed structure is also modular in the horizontal sense, i.e., it grows horizontally whenever a new robot is added to the formation.

Remark 6: the proposed structure is not centralized, since a controller is associated to each robot, except for the three first robots, which are governed by a single controller.

\section{Controllers design}

In this section it is presented the design of the controllers for the following control layers: Formation Control, Kinematic Control and Adaptive Dynamic Compensation. It is worth remark that both the kinematic control and adaptive dynamic compensation are performed separately for each mobile manipulator robot.

\subsection{Formation controller}

The Control Layer receives from the upper layer the desired formation pose and shape $\mathbf{r}_{d}=\left[\begin{array}{ll}\mathbf{P}_{\mathbf{F}_{d}} & \mathbf{S}_{\mathbf{F}_{d}}\end{array}\right]^{T}$ and its desired variations $\dot{\mathbf{r}}_{d}=\left[\begin{array}{ll}\dot{\mathbf{P}}_{\mathbf{F}_{d}} & \dot{\mathbf{S}}_{\mathbf{F}_{d}}\end{array}\right]^{T}$. It generates the pose and shape variation references $\dot{\mathbf{r}}_{\text {ref }}=\left[\begin{array}{ll}\dot{\mathbf{P}}_{\mathbf{F}_{\text {ref }}} & \dot{\mathbf{S}}_{\mathbf{F}_{\text {ref }}}\end{array}\right]^{T}$, where the subscripts $d$ and ref represent the desired and reference signals, respectively. Defining the formation error as $\tilde{\mathbf{r}}(t)=\mathbf{r}_{d}(t)-\mathbf{r}(t)$ and taking its first time derivative, the following expression is obtained,

$$
\dot{\tilde{\mathbf{r}}}=\dot{\mathbf{r}}_{d}-\dot{\mathbf{r}} .
$$


Defining $\tilde{\mathbf{r}}(t)=\mathbf{0}$ as the control objective (an equilibrium point of the system), in order to prove its stability, it is proposed a controller in the sense of Lyapunov as follows. Defining the positive definite candidate function

$$
V(\tilde{\mathbf{r}})=\frac{1}{2} \tilde{\mathbf{r}}^{\mathrm{T}} \tilde{\mathbf{r}}>0,
$$

taking its first time derivative and replacing (6) and $\dot{\mathbf{r}}_{\text {ref }}=\mathrm{J}_{\mathrm{F}} \dot{\mathbf{x}}_{d}$, assuming -by now- perfect velocity tracking, i.e., $\dot{\mathbf{r}} \equiv \dot{\mathbf{r}}_{\text {ref }}$, one gets

$$
\dot{V}(\tilde{\mathbf{r}})=\tilde{\mathbf{r}} \mathbf{T} \dot{\tilde{\mathbf{r}}}=\tilde{\mathbf{r}}^{\mathbf{T}}\left(\dot{\mathbf{r}}_{d}-\mathbf{J}_{\mathbf{F}} \dot{\mathbf{x}}_{d}\right) \cdot
$$

Now, the proposed formation control law is defined as

$$
\dot{\mathbf{x}}_{d}=\mathbf{J}_{\mathbf{F}}^{-\mathbf{1}}\left(\dot{\mathbf{r}}_{d}+K_{1} \tanh \left(K_{2} \tilde{\mathbf{r}}\right)\right)=\mathbf{J}_{\mathbf{F}}^{-1} \dot{\mathbf{r}}_{r e f}
$$

where $k_{1}$ and $k_{2}$ are diagonal positive gain matrix. Introducing (7) into the time derivative of $\dot{V}(\tilde{\mathbf{r}})$, it is obtained

$$
\dot{V}(\tilde{\mathbf{r}})=-\tilde{\mathbf{r}}^{\mathrm{T}} K_{1} \tanh \left(K_{2} \tilde{\mathbf{r}}\right)<0
$$

Thus, the equilibrium point is asymptotically stable, i.e. $\tilde{\mathbf{r}}(t) \rightarrow 0$ asymptotically.

Remark 7: Equation (7) represents the desired reference velocity vector for each mobile manipulator's end-effector.

Now, relaxing the assumption of perfect velocity tracking, it is considered a difference $\delta_{\dot{\mathbf{r}}}(t)$ between the desired and the real formation variations, such as $\boldsymbol{\delta}_{\dot{\mathbf{r}}}=\dot{\mathbf{r}}_{r e f}-\dot{\mathbf{r}}$. Then, (8) should be written as

$$
\dot{V}(\tilde{\mathbf{r}})=\tilde{\mathbf{r}}^{\mathrm{T}} \boldsymbol{\delta}_{\dot{\mathbf{r}}}-\tilde{\mathbf{r}}^{\mathrm{T}} K_{1} \tanh \left(K_{2} \tilde{\mathbf{r}}\right)
$$

A sufficient condition for $\dot{V}(\tilde{\mathbf{r}})$ to be negative definite is,

$$
\left|\tilde{\mathbf{r}}^{\mathrm{T}} K_{1} \tanh \left(K_{2} \tilde{\mathbf{r}}\right)\right|>\left|\tilde{\mathbf{r}}^{\mathrm{T}} \boldsymbol{\delta}_{\dot{\mathbf{r}}}\right|
$$

For large values of $\tilde{\mathbf{r}}$, it can be considered that: $k_{1} \tanh \left(K_{2} \tilde{\mathbf{r}}\right) \approx k_{1} \cdot \dot{V}(\tilde{\mathbf{r}})$ will be negative definite only if: $\left\|K_{1}\right\|>\left\|\boldsymbol{\delta}_{\dot{\mathbf{r}}}\right\|$, thus making the errors $\tilde{\mathbf{r}}$ to decrease. Now, for small values of $\tilde{\mathbf{r}}$, it can be expressed: $K_{1} \tanh \left(K_{2} \tilde{\mathbf{r}}\right) \approx K_{1} K_{2} \tilde{\mathbf{r}}$, and (10) can be written as,

$$
\|\tilde{\mathbf{r}}\|>\frac{\left\|\boldsymbol{\delta}_{\dot{\mathbf{r}}}\right\|}{\lambda_{\text {min }}\left(k_{1}\right) \lambda_{\text {min }}\left(k_{2}\right)}
$$

thus implying that the error $\tilde{\mathbf{r}}$ is bounded by, 


$$
\|\tilde{\mathbf{r}}\| \leq \frac{\left\|\boldsymbol{\delta}_{\dot{\mathbf{r}}}\right\|}{\varsigma \lambda_{\min }\left(k_{1}\right) \lambda_{\min }\left(k_{2}\right)} ; \quad \text { with } 0<\varsigma<1
$$

Hence, with $\boldsymbol{\delta}_{\dot{\mathbf{r}}}(t) \neq \mathbf{0}$, the formation error $\tilde{\mathbf{r}}(t)$ is ultimately bounded by (11).

\subsection{Kinematic controller}

This layer receives the desired position and velocities for each mobile manipulator $\mathbf{x}_{d}=\left[\begin{array}{llllll}\mathbf{h}_{d 1} & \mathbf{h}_{d 2} & \cdots & \mathbf{h}_{d i} & \cdots & \mathbf{h}_{d n}\end{array}\right]^{T}$ and $\dot{\mathbf{x}}_{d}=\left[\begin{array}{llllll}\dot{\mathbf{h}}_{d 1} & \dot{\mathbf{h}}_{d 2} & \cdots & \dot{\mathbf{h}}_{d i} & \cdots & \dot{\mathbf{h}}_{d n}\end{array}\right]^{T}$, respectively, and it generates the desired kinematic velocities $\mathbf{v}_{\mathbf{c}}=\left[\begin{array}{llllll}\mathbf{v}_{\mathbf{c} 1} & \mathbf{v}_{\mathbf{c} 2} & \cdots & \mathbf{v}_{\mathbf{c} i} & \cdots & \mathbf{v}_{\mathbf{c} n}\end{array}\right]^{T}$ for all robots. In other words, the desired operational motion of the $n$ mobile manipulators is an application $\left(\mathbf{x}_{d}(t) \mid t \in\left[t_{0}, t_{f}\right]\right)$. Thus, the problem of control is to find the control vector of maneuverability $\left(\mathbf{v}_{\mathbf{c}}(t) \mid t \in\left[t_{0}, t_{f}\right]\right)$ to achieve the desired operational motion (7). The corresponding evolution of the whole system is given by the actual generalized motion $\left(\mathbf{q}(t) \mid t \in\left[t_{0}, t_{f}\right]\right)$.

The design of the kinematic controller is based on the kinematic model of each mobile manipulator robot that belongs to the work team. The kinematic model (1) of the whole mobile manipulators can be represented by,

$$
\dot{\mathbf{x}}(t)=\mathbf{J}(\mathbf{q}) \mathbf{v}(t)
$$

with

$$
\begin{gathered}
\dot{\mathbf{x}}(t)=\left[\begin{array}{llllll}
\dot{\mathbf{h}}_{1}(t) & \dot{\mathbf{h}}_{2}(t) & \cdots & \dot{\mathbf{h}}_{i}(t) & \cdots & \dot{\mathbf{h}}_{n}(t)
\end{array}\right]^{T} \in \mathfrak{R}^{3 . n}, \\
\mathbf{v}(t)=\left[\begin{array}{llllll}
\mathbf{v}_{1}(t) & \mathbf{v}_{2}(t) & \cdots & \mathbf{v}_{i}(t) & \cdots & \mathbf{v}_{n}(t)
\end{array}\right]^{T} \in \mathfrak{R}^{n \cdot \delta_{n^{\prime}}}, \\
\mathbf{q}(t)=\left[\begin{array}{llllll}
\mathbf{q}_{1}(t) & \mathbf{q}_{2}(t) & \cdots & \mathbf{q}_{i}(t) & \cdots & \mathbf{q}_{n}(t)
\end{array}\right]^{T} \in \mathfrak{R}^{n \cdot n^{\prime}}, \text { and finally } \\
\mathbf{J}(\mathbf{q})=\left[\begin{array}{llllll}
\mathbf{J}_{1}\left(\mathbf{q}_{1}\right) & \mathbf{J}_{2}\left(\mathbf{q}_{3}\right) & \cdots & \mathbf{J}_{i}\left(\mathbf{q}_{i}\right) & \cdots & \mathbf{J}_{n}\left(\mathbf{q}_{n}\right)
\end{array}\right]^{T} \in \mathfrak{R}^{\left(3 . n \times n \cdot n^{\prime}\right)}
\end{gathered}
$$

where $n^{\prime}$ represents the dimensions of the generalized spaces associated to the robotic arms and to the mobile platforms of all mobile manipulators; i.e., $n^{\prime}=n_{1}^{\prime}+n_{2}^{\prime} \ldots+n_{i}^{\prime} \ldots+n_{n}^{\prime}$ (see Remark 2).

It is worth noting, that the kinematic controller is performed separately for each robot. Hence, to obtain the vector of maneuverability $\mathbf{v}_{i}(t)$ that correspond to the $i$-th mobile manipulator, the right pseudo-inverse Jacobian matrix $\mathbf{J}_{i}\left(\mathbf{q}_{i}\right)$ is used

$$
\mathbf{v}_{i}=\mathbf{J}_{i}^{\#} \dot{\mathbf{h}}_{i}
$$


where, $\mathbf{J}_{i}^{\#}=\mathbf{W}_{i}^{-i} \mathbf{J}_{i}^{T}\left(\mathbf{J}_{i} \mathbf{W}_{i}^{-1} \mathbf{J}_{i}^{T}\right)^{-1}$, being $\mathbf{W}_{i}$ a definite positive matrix that weighs the control actions of the system,

$$
\mathbf{v}_{i}=\mathbf{W}_{i}^{-i} \mathbf{J}_{i}^{T}\left(\mathbf{J}_{i} \mathbf{W}_{i}^{-1} \mathbf{J}_{i}^{T}\right)^{-1} \dot{\mathbf{h}}_{i}
$$

The following control law is proposed for the $i$-th mobile manipulator. It is based on a minimal norm solution, which means that, at any time, the mobile manipulator will attain its navigation target with the smallest number of possible movements,

$$
\mathbf{v}_{\mathbf{c} i}=\mathbf{J}_{i}^{\#}\left(\dot{\mathbf{h}}_{\mathbf{d}_{i}}+\mathbf{L}_{\mathbf{K}_{i}} \tanh \left(\mathbf{L}_{\mathbf{K}_{i}}^{\mathbf{1}} \mathbf{K}_{i} \tilde{\mathbf{h}}_{i}\right)\right)+\left(\mathbf{I}_{i}-\mathbf{J}_{i}^{\#} \mathbf{J}_{i}\right) \mathbf{L}_{\mathbf{D}_{i}} \tanh \left(\mathbf{L}_{\mathbf{D}_{i}}^{\mathbf{- 1}} \mathbf{D}_{i} \Lambda_{i}\right)
$$

where, $\dot{\mathbf{h}}_{\mathbf{d}_{i}}=\left[\begin{array}{lll}\dot{h}_{x d} & \dot{h}_{y d} & \dot{h}_{z d}\end{array}\right]^{T}$ is the desired velocities vector of the end-effector, $\tilde{\mathbf{h}}_{i}$ is the control errors vector defined by $\tilde{\mathbf{h}}_{i}=\left[\mathbf{h}_{d i}-\mathbf{h}_{i}\right], \mathbf{K}_{i}, \mathbf{D}_{i}, \mathbf{L}_{\mathbf{K}_{i}}$ and $\mathbf{L}_{\mathbf{D}_{i}}$ are positive definite diagonal gain matrices that weigh the error vector $\tilde{\mathbf{h}}_{i}$ and vector $\Lambda_{i}$. The first term of the right hand side in (13) describes the primary task of the end effector which minimizes $\left\|\mathbf{v}_{i}-\mathbf{J}_{i}^{\#} \dot{\mathbf{h}}_{i}\right\|$. The second term defines self motion of the mobile manipulator in which the matrix $\left(\mathbf{I}_{i}-\mathbf{J}_{i}^{\#} \mathbf{J}_{i}\right)$ projects an arbitrary vector $\Lambda_{i}$ onto the null space of the manipulator Jacobian $\mathcal{N}\left(\mathbf{J}_{i}\right)$ such that the secondary control objectives not affect the primary task of the end-effector. Therefore, any value given to $\Lambda_{i}$ will have effects on the internal structure of the manipulator only, and will not affect the final control of the end-effector at all. By using this term, different secondary control objectives can be achieved effectively, as described in the next subsection.

In order to include an analytical saturation of velocities in the $i$-th mobile manipulator, the $\tanh ($.$) function, which limits the error in \tilde{\mathbf{h}}_{i}$ and the magnitude of the vector $\Lambda_{i}$, is proposed. The expressions $\tanh \left(\mathbf{L}_{\mathbf{K}_{i}}^{\mathbf{- 1}} \mathbf{K}_{i} \tilde{\mathbf{h}}_{i}\right)$ and $\tanh \left(\mathbf{L}_{\mathbf{D}_{i}}^{-\mathbf{1}} \mathbf{D}_{i} \Lambda_{i}\right)$ denote a component by component operation.

On the other hand, the behaviour of the control error of the $i$-th end-effector $\tilde{\mathbf{h}}_{i}$ is now analysed assuming -by now- perfect velocity tracking $\mathbf{v}_{i} \equiv \mathbf{v}_{\mathbf{c} i}$. By substituting (13) in (12) it is obtained

$$
\dot{\tilde{\mathbf{h}}}_{i}+\mathbf{L}_{\mathbf{K}_{i}} \tanh \left(\mathbf{L}_{\mathbf{K}_{i}^{-1}} \mathbf{K}_{i} \tilde{\mathbf{h}}_{i}\right)=\mathbf{0} .
$$

For the stability analysis the following Lyapunov candidate function is considered $V\left(\tilde{\mathbf{h}}_{i}\right)=\frac{1}{2} \tilde{\mathbf{h}}_{i}{ }^{\mathrm{T}} \tilde{\mathbf{h}}_{i}>0$. Its time derivative on the trajectories of the system is

$$
\dot{V}\left(\tilde{\mathbf{h}}_{i}\right)=-\tilde{\mathbf{h}}_{i}{ }^{\mathrm{T}} \mathbf{L}_{\mathbf{K}_{i}} \tanh \left(\mathbf{L}_{\mathbf{K}_{i}}^{-1} \mathbf{K}_{i} \tilde{\mathbf{h}}_{i}\right)<0,
$$


which implies that the equilibrium point of the closed-loop (14) is asymptotically stable, thus the position error of the $i$-th end-effector verifies $\tilde{\mathbf{h}}_{i}(t) \rightarrow \mathbf{0}$ asymptotically with $t \rightarrow \infty$.

\subsubsection{Secondary control objectives}

A mobile manipulator is defined as redundant because it has more degrees of freedom than required to achieve the desired end-effector motion. The redundancy of such mobile manipulators can be effectively used for the achievement of additional performances such as: avoiding obstacles in the workspace and singular configuration, or to optimize various performance criteria. In this Chapter two different secondary objectives are considered: the avoidance of obstacles by the mobile platform and the singular configuration prevention through the system's manipulability control.

\section{Manipulability}

One of the main requirements for an accurate task execution by the robot is a good manipulability, defined as the robot configuration that maximizes its ability to manipulate a target object. Therefore, one of the secondary objectives of the control is to maintain maximum manipulability of the mobile manipulator during task execution. Manipulability is a concept introduced by Yoshikawa (1985) to measure the ability of a fixed manipulator to move in certain directions. Bayle and Fourquet (2001) present a similar analysis for the manipulability of mobile manipulators and extend the concept of manipulability ellipsoid as the set of all end-effector velocities reachable by robot velocities $\mathbf{v}_{i}$ satisfying $\left\|\mathbf{v}_{i}\right\| \leq 1$ in the Euclidean space. A global representative measure of manipulation ability can be obtained by considering the volume of this ellipsoid which is proportional to the quantity $w$ called the manipulability measure,

$$
w=\sqrt{\operatorname{det}\left(\mathbf{J}_{i}\left(\mathbf{q}_{i}\right) \mathbf{J}_{i}^{T}\left(\mathbf{q}_{i}\right)\right)}
$$

Therefore, the mobile manipulator will have maximum manipulability if its internal configuration is such that maximizes the manipulability measure $w$.

\section{Obstacle Avoidance}

The main idea is to avoid obstacles which maximum height does not interfere with the robotic arm. Therefore the arm can follow the desired path while the mobile platform avoids the obstacle by resourcing to the null space configuration. The angular velocity and the longitudinal velocity of the mobile platform will be affected by a fictitious repulsion force. This force depends on the incidence angle on the obstacle $\alpha$, and the distance $d$ to the obstacle. This way, the following control velocities are proposed:

$$
\begin{gathered}
u_{o b s}=Z^{-1}\left(k_{u o b s}\left(d_{o}-d\right)[\pi / 2-|\alpha|]\right) \\
\omega_{o b s}=Z^{-1}\left(k_{\omega o b s}\left(d_{o}-d\right) \operatorname{sign}(\alpha)[\pi / 2-|\alpha|]\right)
\end{gathered}
$$


where, $d_{o}$ is the radius which determines the distance at which the obstacle starts to be avoided, $k_{u o b s}$ and $k_{\omega o b s}$ are positive adjustment gains, the sign function allows defining to which side the obstacle is to be avoided being $\operatorname{sign}(0)=1$. $\mathrm{Z}$ represents the mechanical impedance characterizing the robot-environment interaction, which is calculated as $\mathrm{Z}=I s^{2}+B s+K$ with $I, B$ and $K$ being positive constants representing, respectively, the effect of the inertia, the damping and the elasticity. The closer the platform is to the obstacle, the bigger the values of $\omega_{o b s}$ and $u_{o b s}$.

Taking into account the maximum manipulability (15) and the obstacle avoidance (16) and (17), the vector $\Lambda_{i}$ is now defined as,

$$
\Lambda_{i}=\left[\begin{array}{llllll}
-u_{i o b s} & \omega_{i o b s} & k_{v i 1}\left(\theta_{i 1 d}-\theta_{i 1}\right) & k_{\text {vi } 2}\left(\theta_{i 2 d}-\theta_{i 2}\right) & \ldots & k_{\text {vina }}\left(\theta_{\text {inad }}-\theta_{\text {ina }}\right)
\end{array}\right]^{T}
$$

where $k_{v i}\left(\theta_{i d}-\theta_{i}\right)$ - being $i=1,2, . ., n_{a}$ and $k_{v i}>0$ - are joint velocities proportional to the configuration errors of the mobile robotic arm, in such a way that the manipulator joints will be pulled to the desired $\theta_{i d}$ values that maximize manipulability.

\subsection{Adaptive dynamic compensation controller}

The objective of the Adaptive Dynamic Compensation layer is to compensate for the dynamics of each mobile manipulator, thus reducing the velocity tracking error. This layer receives the desired velocities $\mathbf{v}_{\mathbf{c}}=\left[\begin{array}{llllll}\mathbf{v}_{\mathbf{c} 1} & \mathbf{v}_{\mathbf{c} 2} & \cdots & \mathbf{v}_{\mathbf{c} i} & \cdots & \mathbf{v}_{\mathbf{c} n}\end{array}\right]^{T}$ for all robots, and generates velocity references $\mathbf{v}_{\text {ref }}=\left[\begin{array}{lllllll}\mathbf{v}_{\text {ref } 1} & \mathbf{v}_{\text {ref } 2} & \cdots & \mathbf{v}_{\text {ref } i} & \cdots & \mathbf{v}_{\text {ref } n}\end{array}\right]^{T}$ to be sent to the mobile manipulators.

The adaptive dynamic compensation is performed separately for each robot. Each one of the controllers receive the velocities references $\mathbf{v}_{\mathbf{c} i}(t)$ from the Kinemtic Control layer, and generates another velocities commands $\mathbf{v}_{\text {ref } i}(t)$ for the servos of the $i$-th mobile manipulator.

Thus, if there is no perfect velocity tracking of the $i$-th mobile manipulator, as assumed in Subsection 6.2, the will be a velocity error $\tilde{\mathbf{v}}_{i}(t)$ defined as, $\tilde{\mathbf{v}}_{i}=\mathbf{v}_{\mathbf{c} i}-\mathbf{v}_{i}$ this velocity error motivates to design an adaptive dynamic compensation controller with a robust parameter updating law. It is consider the exact model of the $i$-th mobile manipulator without including disturbances (3),

$$
\mathbf{M}_{i} \dot{\mathbf{v}}_{i}+\mathrm{C}_{i} \mathbf{v}_{i}+\mathbf{G}_{i}=\mathbf{v}_{\text {ref } i}
$$

Hence, the following control law is proposed for the $i$-th mobile manipulator is,

$$
\mathbf{v}_{\text {ref } i}=\Phi_{i} \hat{X}_{i}=\Phi_{i} X_{i}+\Phi_{i} \tilde{X}_{i}=M_{i} \sigma_{i}+C_{i} \mathbf{v}_{\mathbf{c} i}+\mathbf{G}_{i}+\Phi_{i} \tilde{X}_{i}
$$

where $\boldsymbol{\Phi}(\mathbf{q}, \mathbf{v}, \mathbf{\sigma}) \in \mathfrak{R}^{\delta_{n i} \times}, \quad \boldsymbol{\chi}_{i}=\left[\begin{array}{llll}\chi_{1} & \chi_{2} & \cdots & \chi_{l}\end{array}\right]$ and $\hat{\boldsymbol{X}}_{i}=\left[\begin{array}{llll}\hat{\chi}_{1} & \hat{\chi}_{2} & \ldots & \hat{\chi}_{l}\end{array}\right]$ are respectively the unknown vector, real parameters vector and estimated parameters vector of the i-th 
mobile manipulator, whereas $\tilde{X}_{i}=\hat{\chi}_{i}-\chi_{i}$ represents the vector of parameter errors and $\mathbf{\sigma}_{i}=\dot{\mathbf{v}}_{\mathbf{c} i}+\mathbf{L}_{\mathbf{v} i} \tanh \left(\mathbf{L}_{\mathbf{v} i}^{-1} \mathbf{K}_{\mathbf{v} i} \tilde{\mathbf{v}}_{i}\right)$.

In order to obtain the closed-loop equation for the inverse dynamics with uncertain model (19) is equated to (20),

$$
\begin{gathered}
\mathbf{M}_{i} \dot{\mathbf{v}}_{i}+\mathrm{C}_{i} \mathbf{v}_{i}+\mathbf{G}_{i}=\mathbf{M}_{i} \sigma_{i}+\mathbf{C}_{i} \mathbf{v}_{\mathbf{c} i}+\mathbf{G}_{i}+\Phi_{i} \tilde{X}_{i} \\
\mathbf{M}_{i}\left(\sigma_{i}-\dot{\mathbf{v}}_{i}\right)=-\mathbf{C}_{i} \tilde{\mathbf{v}}_{i}-\Phi_{i} \tilde{X}_{i}
\end{gathered}
$$

and next, $\boldsymbol{\sigma}_{i}$ is introduced in (21)

$$
\dot{\tilde{\mathbf{v}}}_{i}=-\mathbf{M}_{i}^{-1} \boldsymbol{\Phi}_{i} \tilde{\boldsymbol{X}}_{i}-\mathbf{M}_{i}^{-1} \mathbf{C}_{i} \tilde{\mathbf{v}}_{i}-\mathbf{L}_{\mathbf{v} i} \tanh \left(\mathbf{L}_{\mathbf{v} i}^{-1} \mathbf{K}_{\mathbf{v} i} \tilde{\mathbf{v}}_{i}\right)
$$

A Lyapunov candidate function is proposed as

$$
V\left(\tilde{\mathbf{v}}_{i}, \tilde{\boldsymbol{X}}_{i}\right)=\frac{1}{2} \tilde{\mathbf{v}}_{i}^{\mathrm{T}} \mathbf{H}_{i} \mathbf{M}_{i} \tilde{\mathbf{v}}_{i}+\frac{1}{2} \tilde{\boldsymbol{X}}_{i}^{\mathrm{T}} \boldsymbol{Y}_{i} \tilde{\boldsymbol{X}}_{i}
$$

where $\mathbf{\gamma}_{i} \in \mathfrak{R}^{l x l}$ is a positive definite diagonal matrix and $\mathbf{H}_{i} \mathbf{M}_{i}$ is a symmetric and positive definite matrix. The time derivative of the Lyapunov candidate function on the system's trajectories is,

$$
\dot{V}\left(\tilde{\mathbf{v}}_{i}, \tilde{\mathbf{X}}_{i}\right)=-\tilde{\mathbf{v}}_{i}^{\mathrm{T}} \mathbf{H}_{i} \mathbf{M}_{i} \mathbf{L}_{\mathbf{v} i} \tanh \left(\mathbf{L}_{\mathbf{v} i}^{-\mathbf{1}} \mathbf{K}_{\mathbf{v} i} \tilde{\mathbf{v}}_{i}\right)-\tilde{\mathbf{v}}_{i}^{\mathrm{T}} \mathbf{H}_{i} \mathbf{C}_{i} \tilde{\mathbf{v}}_{i}-\tilde{\mathbf{v}}_{i}^{\mathrm{T}} \mathbf{H}_{i} \boldsymbol{\Phi}_{i} \tilde{\mathbf{X}}_{i}+\tilde{\boldsymbol{X}}_{i}^{\mathrm{T}} \mathbf{Y}_{i} \dot{\tilde{X}}_{i}+\frac{1}{2} \tilde{\mathbf{v}}_{i}^{\mathrm{T}} \mathbf{H}_{i} \dot{\mathbf{M}}_{i} \tilde{\mathbf{v}}_{i}
$$

Now, recalling that $\mathbf{M}_{i}\left(\mathbf{q}_{i}\right)=\mathbf{H}_{i}^{-1}\left(\overline{\mathbf{M}}_{i}+\mathbf{D}_{i}\right)$ and $\mathbf{C}_{i}\left(\mathbf{q}_{i}, \mathbf{v}_{i}\right)=\mathbf{H}_{i}^{-1}\left(\overline{\mathbf{C}}_{i}+\mathbf{P}_{i}\right)$,

$$
\dot{V}\left(\tilde{\mathbf{v}}_{i}, \tilde{\mathbf{X}}_{i}\right)=-\tilde{\mathbf{v}}_{i}^{\mathbf{T}} \mathbf{H}_{i} \mathbf{M}_{i} \mathbf{L}_{\mathbf{v} i} \tanh \left(\mathbf{L}_{\mathbf{v} i}^{-\mathbf{1}} \mathbf{K}_{\mathbf{v} i} \tilde{\mathbf{v}}_{i}\right)-\tilde{\mathbf{v}}_{i}^{\mathrm{T}}\left(\overline{\mathbf{C}}_{i}+\mathbf{P}_{i}\right) \tilde{\mathbf{v}}_{i}-\tilde{\mathbf{v}}_{i}^{\mathrm{T}} \mathbf{H}_{i} \boldsymbol{\Phi}_{i} \tilde{X}_{i}+\tilde{\boldsymbol{X}}_{i}^{\mathrm{T}} \mathbf{Y}_{i} \dot{\tilde{X}}_{i}+\frac{1}{2} \tilde{\mathbf{v}}_{i}^{\mathrm{T}} \dot{\overline{\mathbf{M}}} \tilde{\mathbf{v}}_{i}
$$

Due to the well known skew-symmetric property of $\left(\dot{\overline{\mathbf{M}}}_{i}-2 \overline{\mathbf{C}}_{i}\right), \dot{V}\left(\tilde{\mathbf{v}}_{i}, \tilde{\mathbf{X}}_{i}\right)$ reduces to,

$$
\dot{V}\left(\tilde{\mathbf{v}}_{i}, \tilde{\mathbf{X}}_{i}\right)=-\tilde{\mathbf{v}}_{i}^{\mathbf{T}} \mathbf{H}_{i} \mathbf{M}_{i} \mathbf{L}_{\mathbf{v} i} \tanh \left(\mathbf{L}_{\mathbf{v} i}^{-1} \mathbf{K}_{\mathbf{v} i} \tilde{\mathbf{v}}_{i}\right)-\tilde{\mathbf{v}}_{i}^{\mathrm{T}} \mathbf{P}_{i} \tilde{\mathbf{v}}_{i}-\tilde{\mathbf{v}}_{i}^{\mathrm{T}} \mathbf{H}_{i} \boldsymbol{\Phi}_{i} \tilde{X}_{i}+\tilde{\mathbf{X}}_{i}^{\mathrm{T}} \mathbf{Y}_{i} \dot{\tilde{X}}_{i}
$$

The following parameter-updating law is proposed for the adaptive dynamic compensation controller. It is based on a leakage term, or $\sigma$ - modification (Kaufman et al., 1998; Sastry and Bodson, 1989). Reference (Nasisi and Carelli, 2003) presented an adaptive visual servo controller with $\sigma$-modification applied to a robot manipulator. By including such term, the robust updating law is obtained

$$
\dot{\hat{X}}_{i}=\gamma_{i}^{-1} \Phi_{i}^{\mathrm{T}} \mathbf{H}_{i} \tilde{\mathbf{v}}_{i}-\boldsymbol{\gamma}_{i}^{-1} \boldsymbol{\Gamma}_{i} \hat{X}_{i}
$$

where $\Gamma_{i} \in \mathfrak{R}^{l \times l}$ is a diagonal positive gain matrix. Equation (25) is rewritten as

$$
\dot{\hat{X}}_{i}=\gamma_{i}^{-1} \Phi_{i}^{\mathrm{T}} \mathbf{H}_{i} \tilde{\mathbf{v}}_{i}-\boldsymbol{\gamma}_{i}^{-1} \Gamma_{i} \tilde{X}_{i}-\boldsymbol{\gamma}_{i}^{-1} \Gamma_{i} \chi_{i}
$$


Let us consider that the dynamic parameters can vary, i.e., $\chi_{i}=\chi_{i}(t)$ and $\dot{\tilde{X}}_{i}=\dot{\hat{X}}_{i}-\dot{X}_{i}$. Substituting (23) in (24),

$$
\dot{V}\left(\tilde{\mathbf{v}}_{i}, \tilde{\mathbf{X}}_{i}\right)=-\tilde{\mathbf{v}}_{i}^{\mathrm{T}} \mathbf{H}_{i} \mathbf{M}_{i} \mathbf{L}_{\mathbf{v} i} \tanh \left(\mathbf{L}_{\mathbf{v} i}^{-1} \mathbf{K}_{\mathbf{v} i} \tilde{\mathbf{v}}_{i}\right)-\tilde{\mathbf{v}}_{i}^{\mathrm{T}} \mathbf{P}_{i} \tilde{\mathbf{v}}_{i}-\tilde{\boldsymbol{X}}_{i}^{\mathrm{T}} \boldsymbol{\Gamma}_{i} \tilde{\mathbf{X}}_{i}-\tilde{\boldsymbol{X}}_{i}^{\mathrm{T}} \boldsymbol{\Gamma}_{i} \boldsymbol{X}_{i}-\tilde{\boldsymbol{X}}_{i}^{\mathrm{T}} \mathbf{V}_{i} \dot{\boldsymbol{X}}_{i}
$$

Considering small values of $\tilde{\mathbf{v}}_{i}$, then $\mathbf{L}_{\mathbf{v} i} \tanh \left(\mathbf{L}_{\mathbf{v} i}^{-1} \mathbf{K}_{\mathbf{v} i} \tilde{\mathbf{v}}_{i}\right) \approx \mathbf{K}_{\mathbf{v} i} \tilde{\mathbf{v}}_{i}$. The following constants are defined: $v_{\Gamma}=k_{\max }\left(\boldsymbol{\Gamma}_{i}\right), \quad v_{\gamma}=k_{\max }\left(\mathbf{\gamma}_{i}\right), \mu_{\Gamma}=\chi\left(\boldsymbol{\Gamma}_{i}\right), \quad \mu_{M K_{v} P}=\chi_{i}\left(\mathbf{H}_{i} \mathbf{M}_{i} \mathbf{K}_{\mathbf{v} i}\right)+\chi_{i}\left(\mathbf{P}_{i}\right)$, where $\chi(\mathbf{Z})=\sqrt{\lambda_{\min }\left(\mathbf{Z}^{\mathrm{T}} \mathbf{Z}\right)}$ is the minimum singular value to $\mathbf{Z}, k_{\max }(\mathbf{Z})=\sqrt{\lambda_{\max }\left(\mathbf{Z}^{\mathrm{T}} \mathbf{Z}\right)}$ denotes the maximum singular value of $\mathbf{Z}$, and $\lambda_{\min }($.$) and \lambda_{\max }($.$) represent the smallest and the$ biggest eigenvalues of a matrix, respectively. Then, $\dot{V}$ can be rewritten as,

$$
\dot{V}\left(\tilde{\mathbf{v}}_{i}, \tilde{X}_{i}\right)=-\mu_{M K_{v} P}\left\|\tilde{\mathbf{v}}_{i}\right\|^{2}-\mu_{\Gamma}\left\|\tilde{X}_{i}\right\|^{2}+v_{\Gamma}\left\|\tilde{X}_{i}\right\|\left\|X_{i}\right\|+v_{\gamma}\left\|\tilde{X}_{i}\right\|\left\|\dot{X}_{i}\right\| .
$$

Considering $\zeta \in \mathfrak{R}^{+}$in the difference square,

$$
\left(\frac{1}{\zeta}\left\|\tilde{X}_{i}\right\|-\zeta\left\|x_{i}\right\|\right)^{2}=\frac{1}{\zeta}\left\|\tilde{X}_{i}\right\|^{2}-2\left\|\tilde{X}_{i}\right\|\left\|x_{i}\right\|+\zeta^{2}\left\|x_{i}\right\|^{2}
$$

can be written as,

$$
\left\|\tilde{X}_{i}\right\|\left\|x_{i}\right\| \leq \frac{1}{2 \zeta^{2}}\left\|\tilde{X}_{i}\right\|^{2}+\frac{\zeta^{2}}{2}\left\|x_{i}\right\|^{2}
$$

By applying a similar reasoning with $\eta \in \mathfrak{R}^{+}$, it can be obtained

$$
\left\|\tilde{X}_{i}\right\|\left\|\dot{X}_{i}\right\| \leq \frac{1}{2 \eta^{2}}\left\|\tilde{X}_{i}\right\|^{2}+\frac{\eta^{2}}{2}\left\|\dot{X}_{i}\right\|^{2}
$$

Substituting (30) and (29) in (28)

$$
\dot{V}\left(\tilde{\mathbf{v}}_{i}, \tilde{X}_{i}\right) \leq-\mu_{M K_{v} P}\left\|\tilde{\mathbf{v}}_{i}\right\|^{2}-\mu_{\Gamma}\left\|\tilde{X}_{i}\right\|^{2}+v_{\Gamma}\left(\frac{1}{2 \zeta^{2}}\left\|\tilde{X}_{i}\right\|^{2}+\frac{\zeta^{2}}{2}\left\|X_{i}\right\|^{2}\right)+v_{\gamma}\left(\frac{1}{2 \eta^{2}}\left\|\tilde{X}_{i}\right\|^{2}+\frac{\eta^{2}}{2}\left\|\dot{X}_{i}\right\|^{2}\right)
$$

Equation (31) can be written in compact form as

$$
\dot{V}\left(\tilde{\mathbf{v}}_{i}, \tilde{\mathbf{X}}_{i}\right) \leq-\varepsilon_{1}\left\|\tilde{\mathbf{v}}_{i}\right\|^{2}-\varepsilon_{2}\left\|\tilde{\mathbf{X}}_{i}\right\|^{2}+\delta
$$

where, $\varepsilon_{1}=\mu_{M K_{v} P}>0, \varepsilon_{2}=\mu_{\Gamma}-\frac{v_{\Gamma}}{2 \zeta^{2}}-\frac{v_{\gamma}}{2 \eta^{2}}>0$ and $\delta=v_{\Gamma} \frac{\zeta^{2}}{2}\left\|X_{i}\right\|^{2}+v_{\gamma} \frac{\eta^{2}}{2}\left\|\dot{X}_{i}\right\|^{2}$, with $\zeta$ and $\eta$ conveniently selected. Now, from the Lyapunov candidate function $V\left(\tilde{\mathbf{v}}_{i}, \tilde{\mathbf{X}}_{i}\right)=\frac{1}{2} \tilde{\mathbf{v}}_{i}^{\mathrm{T}} \mathbf{H}_{i} \mathbf{M} \tilde{\mathbf{v}}_{i}+\frac{1}{2} \tilde{\boldsymbol{X}}_{i}^{\mathrm{T}} \mathbf{Y}_{i} \tilde{X}_{i}$ it can be stated that 


$$
V \leq \beta_{1}\left\|\tilde{\mathbf{v}}_{i}\right\|^{2}+\beta_{2}\left\|\tilde{X}_{i}\right\|^{2}
$$

where $\beta_{1}=\frac{1}{2} \vartheta_{\bar{M}}, \beta_{2}=\frac{1}{2} \vartheta_{\gamma}, \vartheta_{\bar{M}}=k_{\max }\left(\mathbf{H}_{i} \mathbf{M}_{i}\right), \vartheta_{\gamma}=k_{\max }\left(\mathbf{\gamma}_{i}\right)$. Then,

$$
\dot{V} \leq-\Lambda V+\delta
$$

with $\Lambda=\left\{\frac{\varepsilon_{1}}{\beta_{1}}, \frac{\varepsilon_{2}}{\beta_{2}}\right\}$. Since $\delta$ is bounded, (34) implies that $\tilde{\mathbf{v}}_{i}(t)$ and $\tilde{X}_{i}(t)$ are finally bounded. Therefore, the $\sigma$-modification term makes the adaptation law more robust at the expense of increasing the error bound. As $\delta$ is a function of the minimum singular value of the gain matrix $\Gamma_{i}$ of the $\sigma$-modification term, and its values are arbitrary, then the error bound can be made small. Note that the proposed adaptive dynamic controller does not guarantee that $\tilde{X}_{i}(t) \rightarrow 0$ as $t \rightarrow \infty$. In other words, estimated parameters might converge to values that are different from their true values. Actually, it is not required that $\tilde{X}_{i}(t) \rightarrow 0$ in order to make $\tilde{\mathbf{v}}_{i}(t)$ converge to a bounded value.

Remark 8: Note that the updating law (25) needs the $\mathbf{H}_{i}$ matrix. This matrix includes parameters of the actuators, which can be easily known and remain constant. Therefore, this is not a relevant constraint within the adaptive control design.

\subsection{Stability analysis considering $\tilde{\mathbf{h}}_{i}(t)$ and $\tilde{\mathbf{v}}_{i}(t)$}

The behaviour of the tracking error of the end-effector $\mathbf{h}_{i}(t)$ of the $i$-th mobile manipulator is now analysed relaxing the assumption of perfect velocity tracking. Therefore, the (14) is now written as,

$$
\dot{\tilde{\mathbf{h}}}_{i}+\mathbf{L}_{\mathbf{K} i} \tanh \left(\mathbf{L}_{\mathbf{K} i}^{-1} \mathbf{K}_{i} \tilde{\mathbf{h}}_{i}\right)=\mathbf{J}_{i} \tilde{\mathbf{v}}_{i}
$$

where, $\tilde{\mathbf{v}}_{i}$ is the velocity error of the $i$-th mobile manipulator and $\mathbf{J}_{i}$ the Jacobian matrix. It is considered a Lyapunov candidate function $V\left(\tilde{\mathbf{h}}_{i}\right)=\frac{1}{2} \tilde{\mathbf{h}}_{i}^{\mathrm{T}} \tilde{\mathbf{h}}_{i}$ and its time derivative,

$$
\dot{V}\left(\tilde{\mathbf{h}}_{i}\right)=\tilde{\mathbf{h}}_{i}^{\mathbf{T}} \mathbf{J}_{i} \tilde{\mathbf{v}}_{i}-\tilde{\mathbf{h}}_{i}^{\mathrm{T}} \mathbf{L}_{\mathbf{K} i} \tanh \left(\mathbf{L}_{\mathbf{K} i}^{-1} \mathbf{K}_{i} \tilde{\mathbf{h}}_{i}\right) .
$$

A sufficient condition for $\dot{V}\left(\tilde{\mathbf{h}}_{i}\right)$ to be negative definite is

$$
\left|\tilde{\mathbf{h}}_{i}^{\mathbf{T}} \mathbf{L}_{\mathbf{K} i} \tanh \left(\mathbf{L}_{\mathbf{K} i}^{\mathbf{- 1}} \mathbf{K} \tilde{\mathbf{h}}_{i}\right)\right|>\left|\tilde{\mathbf{h}}_{i}^{\mathrm{T}} \mathbf{J}_{i} \tilde{\mathbf{v}}_{i}\right| .
$$

Following a similar analysis to the one in Section 6.1, it can be concluded that, if $\left\|\mathbf{L}_{\mathbf{K}_{i}}\right\|>\left\|\mathbf{J}_{i}\right\|\left\|\tilde{\mathbf{v}}_{i}\right\|$ thus implying that the error $\tilde{\mathbf{h}}_{i}$ is bounded by, 


$$
\left\|\tilde{\mathbf{h}}_{i}\right\| \leq \frac{\left\|\mathbf{J}_{i} \tilde{\mathbf{v}}_{i}\right\|}{\varsigma \lambda_{\text {min }}\left(\mathbf{K}_{i}\right)} ; \quad \text { with } 0<\varsigma<1
$$

Hence, if $\mathbf{v}_{i} \neq \mathbf{v}_{\mathbf{c} i}$ it is concluded that $\tilde{\mathbf{h}}_{i}(t)$ is ultimately bounded by (37).

Now finally, generalizing (35) for whole multi-layer control scheme, it is obtained

$$
\dot{\tilde{\mathbf{x}}}+\mathbf{L}_{\mathbf{K}} \tanh \left(\mathbf{L}_{\mathbf{K}}^{-1} \mathbf{K} \tilde{\mathbf{x}}\right)=\mathbf{J} \tilde{\mathbf{v}}
$$

where $\dot{\tilde{\mathbf{x}}}=\left[\begin{array}{llllll}\dot{\tilde{\mathbf{h}}}_{1} & \dot{\tilde{\mathbf{h}}}_{2} & \cdots & \dot{\tilde{\mathbf{h}}}_{i} & \cdots & \dot{\tilde{\mathbf{h}}}_{n}\end{array}\right]^{T}$ represents the position error vector of all mobile manipulators, $\mathbf{K}$ and $\mathbf{L}_{\mathbf{K}}$ are positive definite diagonal matrices defined as $\mathbf{K}=\operatorname{diag}\left(\mathbf{K}_{1}, \mathbf{K}_{2} . ., \mathbf{K}_{i} . ., \mathbf{K}_{n}\right)$ and $\mathbf{L}_{\mathbf{K}}=\operatorname{diag}\left(\mathbf{L}_{\mathbf{K} 1}, \mathbf{L}_{\mathbf{K} 2 . .,} \mathbf{L}_{\mathbf{K} i} . ., \mathbf{L}_{\mathbf{K} n}\right)$, respectively, while the function $\tanh ($.$) denote a component by component operation. By applying a similar$ reasoning as in (35), it is can concluded that $\tilde{\mathbf{x}}(t)$ is bounded by,

$$
\|\tilde{\mathbf{x}}\| \leq \frac{\|\mathbf{J} \tilde{\mathbf{v}}\|}{\varsigma \lambda_{\min }(\mathbf{K})} ; \quad \text { with } 0<\varsigma<1
$$

For the case of perfect velocity tracking $\mathbf{v} \equiv \mathbf{v}_{\mathbf{c}}$, i.e., $\tilde{\mathbf{v}} \equiv \mathbf{0}$, it is concluded that $\tilde{\mathbf{x}}(t) \rightarrow \mathbf{0}$, which implies that $\tilde{\mathbf{r}}(t) \rightarrow \mathbf{0}$ asymptotically with $t \rightarrow \infty$, thus accomplishing the control objective. Nevertheless, this is not always possible in real contexts, therefore if it is considered the velocity error $\tilde{\mathbf{v}}(t) \neq \mathbf{0}$, consequently it has that $\tilde{\mathbf{x}}(t) \neq \mathbf{0}$. This implies that the formation error is nonzero $\boldsymbol{\delta}_{\dot{\mathbf{r}}}(t) \neq \mathbf{0}$, i.e., the formation error $\boldsymbol{\delta}_{\dot{\tilde{\mathbf{r}}}}(t)$ is related with the errors $\tilde{\mathbf{x}}(t)$ and $\tilde{\mathbf{v}}(t)$. Therefore from (4), (5), (7) and (38) it is obtained the following error expression,

$$
\boldsymbol{\delta}_{\dot{\mathbf{r}}}(t)=\mathbf{J}_{\mathbf{F}}(\mathbf{x})\left[\mathbf{J}(\mathbf{q}) \tilde{\mathbf{v}}-\mathbf{L}_{\mathbf{K}} \tanh (\tilde{\mathbf{x}})\right]
$$

Thus, it is concluded that the adaptive dynamic compensation reduces the velocity error $\tilde{\mathbf{v}}(t)$ and consequently the error $\tilde{\mathbf{x}}(t)$, hence formation error $\delta_{\dot{\mathbf{r}}}(t)$ is also reduced. Finally, with this results and the conclusion previously obtained from (11), the adaptive dynamic compensation controller reduces the height limit of the formation error $\tilde{\mathbf{r}}(t)$.

\section{Simulation result and discussion}

In order to assess and discuss the performance of the proposed coordinated cooperative controller, it was developed a simulation platform for multi-mobile manipulators with Matlab interface, see the Figure 5. It is important mention that the developed simulator has incorporated the dynamic model of the robot. This is an online simulator, which allows users to view three-dimensional environment navigation of mobile manipulators. Ours simulation platform is based on the MRSiM platform presented by Brandao et al., 2008.

The $i$-th 6 DOF mobile manipulator used in the simulation is shown in Figure 5, which is composed by a non holonomic mobile platform, a laser rangefinder mounted on it, and a 3 DOF robotic arm. In order to illustrate the performance of the proposed multi-layer control 
scheme, several simulations were carried out for coordinated cooperative control of mobile manipulators. Most representative results are presented in this section.

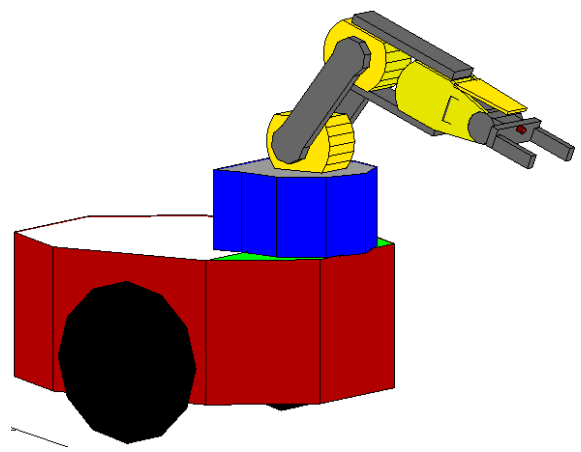

Fig. 5. Mobile manipulator robot used by the simulation platform

The simulation experiments consist of a team of three or more mobile mamipulator tracking a desired trajectory while carrying a payload cooperatively. Also, the obstacle avoidance and the singular configuration prevention through the system's manipulability control are considered in the simulations. It is assumed the existence of several obstacles, which have a maximum height that does not interfere with the robotic arms. That is, the obstacles only affect the platform navigation. Hence, the task for each mobile manipulator is divided into two subtasks. The first subtask: to carry a payload cooperatively; and the second subtask: obstacle avoidance and the singular configuration prevention.

It is important to remark that for all experiments in this section it was considered that there is an error of $30 \%$ in dynamic model parameters of each one mobile manipulator robot.

In the first one it is considered both the position and orientacion of the virtual structure. For this case, the desired positions of the arm joints are, Robot_1: $\theta_{1 d}=0[\mathrm{rad}]$, $\theta_{2 d}=-0.6065[\mathrm{rad}], \theta_{3 d}=1.2346[\mathrm{rad}]$. Robot_2 and Robot_3: $\theta_{1 d}=0[\mathrm{rad}], \theta_{2 d}=0.6065[\mathrm{rad}]$, and $\theta_{3 d}=-1.2346[\mathrm{rad}]$. Also, the desired virtual structure is selected as, $p_{F_{d}}=1.75[\mathrm{~m}]$, $q_{F_{d}}=1.2[\mathrm{~m}]$ and $\beta_{F_{d}}=1.4[\mathrm{rad}], z_{1 F_{d}}=0.4[\mathrm{~m}]$ and $z_{2 F_{d}}=z_{3 F_{d}}=0.3[\mathrm{~m}]$; while the desired trajectory for the prism centroid is described by:

$$
\begin{gathered}
x_{F_{d}}=0.2 t+3.56, \\
y_{F_{d}}=3 \cos (0.1 t)+3 \text { and } \\
\psi_{F_{d}}=\frac{\pi}{2}+\gamma,
\end{gathered}
$$

where $\gamma=\arctan \left(\frac{d y_{F d}}{d t} / \frac{d x_{F d}}{d t}\right)$. It is worth noting that this trajectory was chosen in order to excite the dynamics of the robots by changing their acceleration. The values of the gains matrices were adjusted considering the system performance with the dynamic compensation deactivated. After this gain setting, the values obtained were used in all simulations. 
Figures 6 - 9 show the results of the simulation experiment. Figure 6 shows the stroboscopic movement on the X-Y-Z space. It can be seen that the proposed controller works correctly, where three mobile manipulators work in coordinated and cooperative form while transporting a common object. It can be noticed in Figure 6 that there are three triangles of different colours representing the upper side of a virtual structure. The yellow triangle represents the shape-position of the virtual structure that describes the end-effector of the mobile manipulator robots, while the pink triangle represents the location and shape of the upper side of the desired virtual structure, and the orange triangle indicates that both previously mentioned position-shapes are equal. While, figures $7-9$ show that the control errors $\tilde{\mathbf{r}}(t)$ achieve values close to zero. Figure 7 shows the errors of position and orientation of the virtual structure and in Figures 8 and 9 illustrate the errors of the virtual structure shape.

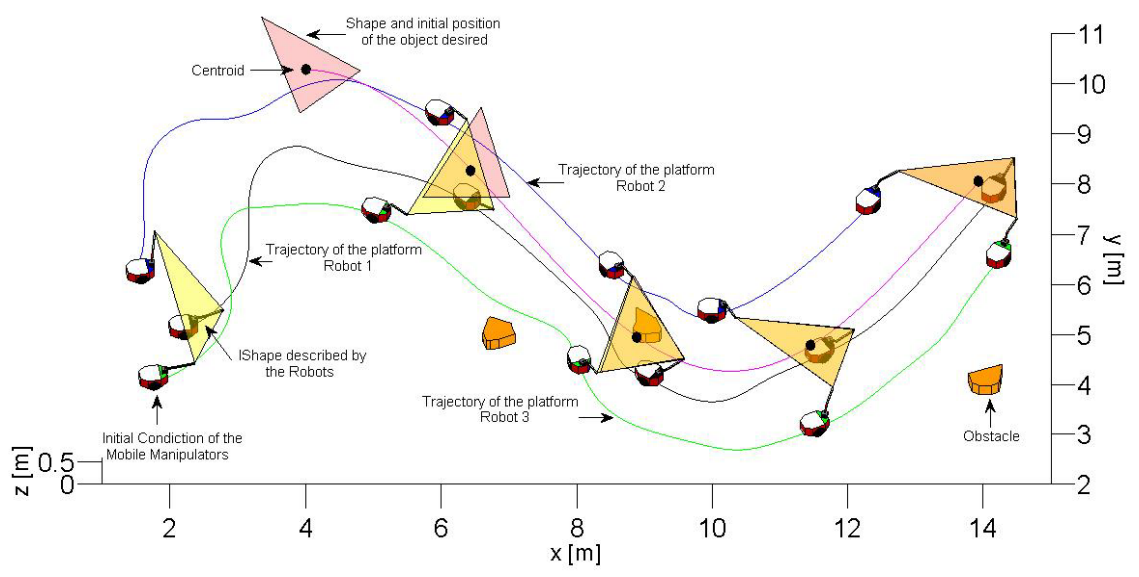

Fig. 6. Cooperative Coordinated Control of Mobile Manipulators

In order to show the scalability of the control structure for $n>3$ mobile manipulators, the following simulations were carried out for coordinated cooperative control of multi-mobile manipulators.

In this context, the second simulation experiment shows a coordinated and cooperative control between four mobile manipulators. In this simulation the robots should navigate while carrying a payload, following a desired previously defined trajectory. It is considered a partially structured environment containing several obstacles in the trajectory. 


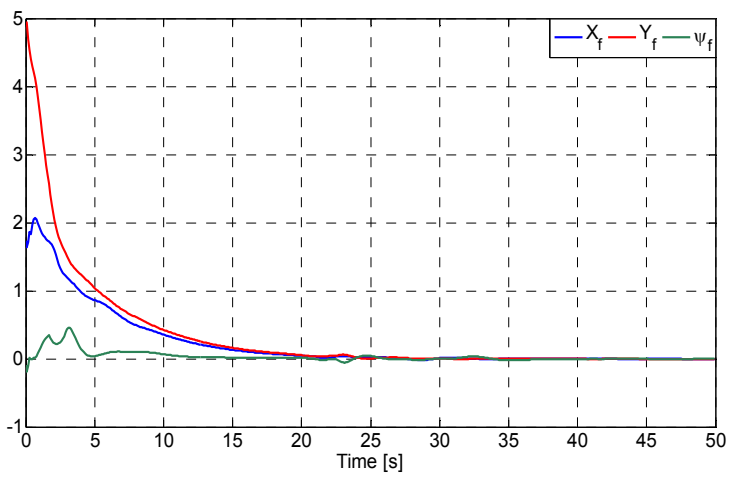

Fig. 7. Position and orientation errors

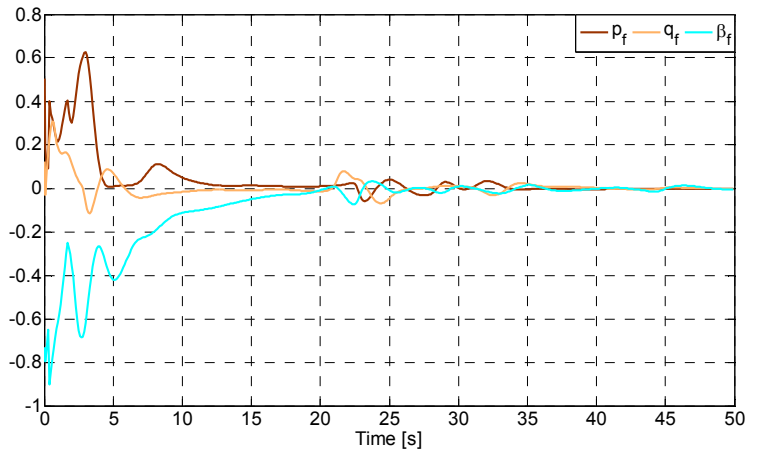

Fig. 8. Structure shape errors

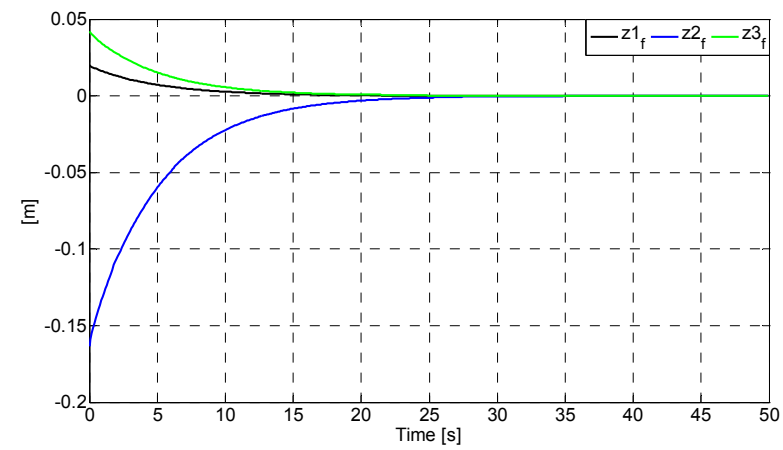

Fig. 9. Structure shape errors (heights)

Figures 10 show the stroboscopic movement on the X-Y-Z space, where it can be seen not only the good performance of the proposed cooperative control, but also the scalability of the proposal for multi-mobile manipulators. The pose and shape errors of the simulation experiments are shown in Figures $11-14$. 


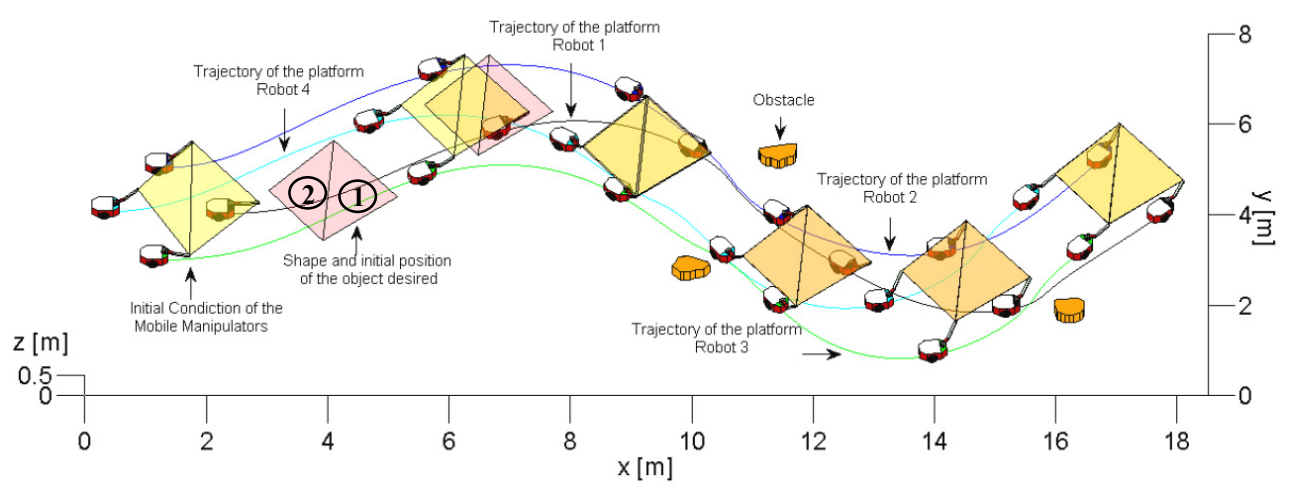

Fig. 10. Cooperative Coordinated Control of Mobile Manipulators

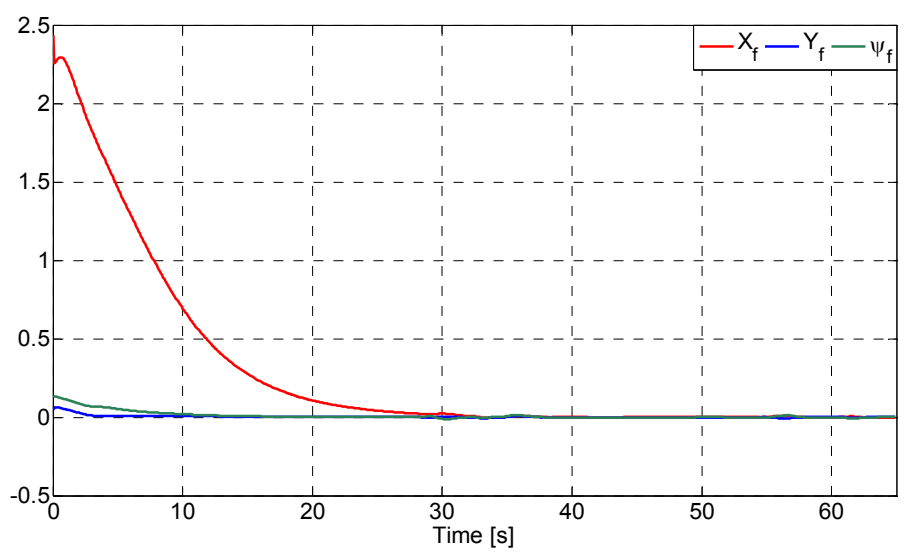

Fig. 11. Position and orientation errors of the triangle(1)

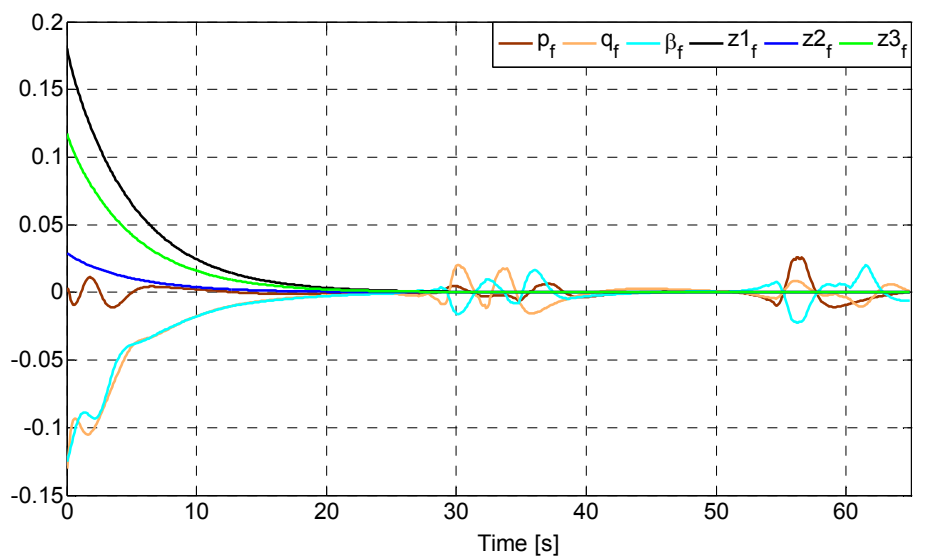

Fig. 12. Structure shape errors of the triangle(1) 


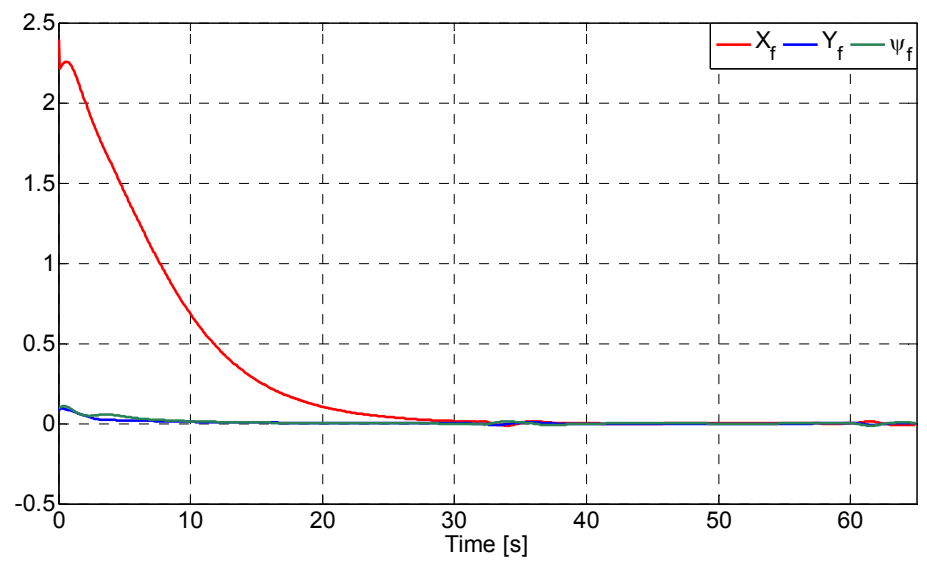

Fig. 13. Position and orientation errors of the triangle (2)

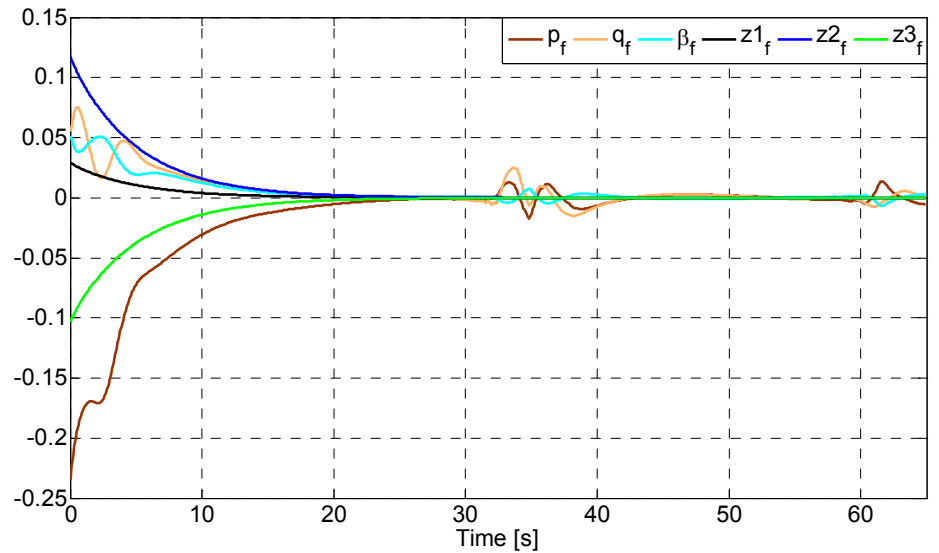

Fig. 14. Structure shape errors of the triangle(2)

\section{Conclusion}

A multi-layer control scheme for adaptive cooperative coordinated control of $n \geq 3$ mobile manipulators, for transporting a common object was presented in this Chapter. Each control layer works as an independent module, dealing with a specific part of the problem of the adaptive coordinated cooperative control. On the other hand, the i-th mobile manipulator redundancy is used for the achievement of secondary objectives such as: the singular configuration prevention through the control of the system's manipulability and the avoidance of obstacles by the mobile platforms. Stability of the system has been analytically proved, concluding that the formation errors are ultimately bounded. The results, which were obtained by simulation, show a good performance of the proposed multi-layer control scheme. 


\section{References}

Andaluz V. Roberti Flavio, Carelli Ricardo. (2010). Robust Control with Redundancy Resolution and Dynamic Compensation for Mobile Manipulators. IEEE-ICIT Internacional Conference on Industrial Technology, pp. 1449-1454, 2010.

Antonelli G., Arrichiello F., and Chiaverini S. (2008). The Entrapment/Escorting Mission. IEEE Robotics \& Automation Magazine, Vol. 15, no. 1, pp. 22-29.

Bayle B. and J.-Y. Fourquet, "Manipulability Analysis for Mobile Manipulators", IEEE International Conference on Robots \& Automation, pp. 1251-1256, 2001.

Bicchi A., Danesi A., Dini G., Porta S., Pallottino L., Savino I., and Schiavi R. (2008). “Heterogeneous Wireless Multirobot System. IEEE Robotics \& Automation Magazine, Vol. 15, no. 1, pp. 62-70.

Brandao A. S., Carelli R., Sarcinelli-Filho M., and BastosFilho T. F. (2008). MRSiM: An environment for simulating mobile robots navigation. Jornadas Argentinas de Rob6tica, (written in Spanish).

Cao Y. U., Fukunaga A. S., and Kahng A. B. (1997). Cooperative mobile robotics: Antecedents and directions. Autonomous Robots, no. 4, pp. 1-23.

Consolini L., Morbidi F., Prattichizzo D., and Tosques M. (2007). A geometric characterization of leader-follower formation control. IEEE International Conference on Robotics and Automation (ICRA07), pp. 2397-2402.

Fujii M., Inamura W., Murakami H. and Tanaka K. (2007). Cooperative Control of Multiple Mobile Robots Transporting a Single Object with Loose Handling. IEEE International Conference on Robotics and Biomimetics. pp. 816-822.

Hao Su and Venkat Krovi. (2008). Decentralized Dynamic Control of a Nonholonomic Mobile Manipulator Collective: a Simulation Study. ASME Dynamic Systems and Control Conference. pp.1-8.

Hirata Y., Kume Y., Sawada T., Wang Z., and Kosuge K. (2004). Handling of an object by multiple mobile manipulators in coordination based on casterlike dynamics. IEEE Int. Conf. Robot. Autom. Vol. 26, pp. 807-812.

Hougen D., Benjaafar S., B. J.C., Budenske J., Dvoraktt M., Gini M., French H., Krantz D., Li P., Malver F., Nelson B., Papanikolopoulos N., Rybski P., Stoeter S., Voyles R., and Yesin K. (2000). A miniature robotic system for reconnaissance and surveillance. IEEE International Conference on Robotics and Automation (ICRA'00), pp. 501-507.

Jennings J. S., Whelan G., and Evans W. F. (1997). Cooperative search and rescue with a team of mobile robots. 8th IEEE-ICAR, pp. 193-200.

Kaufman H., Barkana I. and Sobel K. (1998). Direct adaptive control algorithms, theory and applications. New York, USA: Springer-Verlag.

Khatib O., Yokoi K., Chang K., Ruspini D., Holmberg R., and Casal A. (1996). Vehicle/Arm Coordination and Multiple Mobile Manipulator. Intelligent Robots and Systems, Vol.2 pp. $546-553$.

Martins F. N., Celeste W., Carelli R., Sarcinelli-Filho M., and Bastos-Filho T. (2008). An Adaptive Dynamic Controller for Autonomous Mobile Robot Trajectory Tracking. Control Engineering Practice. Vol. 16, pp. 1354-1363.

Mas I., Petrovic O., and Kitts C. (2008). Cluster space specification and control of a 3-robot mobile system. IEEE International Conference on Robotics and Automation - ICRA. pp. 3763-3768. 
Nasisi O. and Carelli R. (2003). Adaptive servo visual robot control. Robotics and Autonomous Systems, 43 (1), pp. 51-78.

Sastry S., and Bodson M. (1989). Adaptive control-stability, convergence and robustness. Englewood Cliffs, NJ, USA: Prentice-Hall.

Sciavicco, L. and Siciliano, B. (2000). Modeling and Control of Robot Manipulators. Great Britain: Springer-Verlag London Limited.

Spong M. and Vidyasagar M., (1989). Robot Dynamics and Control.

Stouten B. and De Graaf A. (2004). Cooperative transportation of a large object-development of an industrial application. IEEE - ICRA, vol. 3, pp. 2450-2455.

Tanner H. G., Loizou S., and Kyriakopoulos K. J. (2003). Nonholonomic navigation and control of cooperating mobile manipulators. IEEE Trans. Robot. Autom. Vol.19, no. 1, pp. 53-64.

Thomas G. Sugar and Vijay Kumar. (2002). Control of Cooperating Mobile Manipulators. IEEE Transactions on Robotics and Automation. Vol. 18, no. 1, pp. 94-103.

Xin Chen and Yangmin Li. (2006). Cooperative Transportation by Multiple Mobile Manipulators Using Adaptive NN Control. International Joint Conference on Neural Networks. pp. 4193-4200.

Yamamoto Y., Hiyama Y., and Fujita A. (2004). Semi-autonomous reconfiguration of wheeled mobile robots in coordination. IEEE Int. Conf. Robot. Autom., pp. 34563461.

Yasuhisa Hirata, Youhei Kume, Zhi-Dong Wang, Kszuhiro Kosiige. (2003). Coordinated Motion Control of Multiple Mobile Manipulators based on Virtual 3-D Caster. International Conference on Robotics, Intelligent Systcms and Signal Processing. pp. 1924.

Yoshikawa T. (1985). Dynamic manipulability of robot manipulators. International Journal of Robotic Systems, pp. 113-124.

Yoshikawa T., (1990). Foundations of Robotics: Analysis and Control. The MIT Press, Cambridge, MA.

Zhijun Lia, b, Shuzhi Sam Gea, , and Zhuping Wanga. (2008). Robust adaptive control of coordinated multiple mobile manipulators. Mechatronics. Vol. 18, pp. 239-250.

Zhijun Li, Pey Y. Tao, Shuzhi S. Ge, Martin Adams, and Wijerupage S. W. (2009). Robust Adaptive Control of Cooperating Mobile Manipulators With Relative Motion. IEEE Transactions on Systems, Man, and Cybernetics. Part B: Cybernetics, Vol. 39, no. 1, pp. 103-116. 
(C) 2012 The Author(s). Licensee IntechOpen. This is an open access article distributed under the terms of the Creative Commons Attribution 3.0 License, which permits unrestricted use, distribution, and reproduction in any medium, provided the original work is properly cited. 